\title{
Some Aggregation Operators Based on Einstein Operations under Interval-Valued Dual Hesitant Fuzzy Setting and Their Application
}

\author{
Wenkai Zhang, Xia Li, and Yanbing Ju \\ School of Management and Economics, Beijing Institute of Technology, Beijing 100081, China \\ Correspondence should be addressed to Yanbing Ju; juyb@bit.edu.cn
}

Received 28 April 2014; Accepted 7 August 2014; Published 4 November 2014

Academic Editor: Wudhichai Assawinchaichote

Copyright (c) 2014 Wenkai Zhang et al. This is an open access article distributed under the Creative Commons Attribution License, which permits unrestricted use, distribution, and reproduction in any medium, provided the original work is properly cited.

\begin{abstract}
We investigate the multiple attribute decision making (MADM) problems in which attribute values take the form of interval-valued dual hesitant fuzzy information. Firstly, some operational laws for interval-valued dual hesitation fuzzy elements (IVDHFEs) based on Einstein operations are developed. Then we develop some aggregation operators based on Einstein operations: the intervalvalued dual hesitant fuzzy Einstein weighted averaging (IVDHFEWA) operator, interval-valued dual hesitant fuzzy Einstein ordered weighted averaging (IVDHFEOWA) operator, interval-valued dual hesitant fuzzy Einstein hybrid averaging (IVDHFEHA) operator, interval-valued dual hesitant fuzzy Einstein weighted geometric (IVDHFEWG) operator, interval-valued dual hesitant fuzzy Einstein ordered weighted geometric (IVDHFEOWG) operator, and interval-valued dual hesitant fuzzy Einstein hybrid geometric (IVDHFEHG) operator. Furthermore, we discuss some desirable properties of these operators, and investigate the relationship between the developed operators and the existing ones. Based on the IVDHFEWA operator, an approach to MADM problems is proposed under the interval-valued dual hesitant fuzzy environment. Finally, a numerical example is given to show the application of the developed method, and a comparison analysis is conducted to demonstrate the effectiveness of the proposed approach.
\end{abstract}

\section{Introduction}

The fuzzy set [1] has received increasing attention since its introduction by Zadeh. Various extensions of this theory have been developed, including the interval-valued fuzzy set [2], type-2 fuzzy set [3], intuitionistic fuzzy set [4], intervalvalued intuitionistic fuzzy set [5], and linguistic fuzzy set [6]. However, the aforementioned extensions cannot deal with the situation where it is difficult to determine the membership of an element to a set owing to ambiguity among several different values; that is, the difficulty in establishing the membership of an element to a set does not arise from a margin of error (as in intuitionistic or interval-valued fuzzy sets) or a specified possibility distribution of the possible values (as in type-2 fuzzy set) but instead arises from our hesitation among a few different values. Recently, Torra and Narukawa [7] and Torra [8] introduced the concept of hesitant fuzzy sets (HFSs) to handle such cases. HFSs permit the membership degree of an element to a set to be represented by a set of possible values. Hesitant fuzzy aggregation operators have received increasing attention from researchers recently. Xia and $\mathrm{Xu}$ [9] defined some hesitant fuzzy operational rules and discussed a series of operators under various conditions. Furthermore, Xia et al. [10] developed some quasiarithmetic aggregation operators and some induced aggregation operators for hesitant fuzzy information. Zhu et al. [11] defined the hesitant fuzzy geometric Bonferroni mean (HFGBM), the hesitant fuzzy Choquet geometric Bonferroni mean (HFCGBM) and then applied them to MADM problems. Motivated by the idea of prioritized aggregation operators, Wei [12] developed some prioritized aggregation operators for aggregating hesitant fuzzy information. Zhang [13] extended the classical power aggregation operators to hesitant fuzzy environment and then developed the hesitant fuzzy power averaging (HFPA) operator, generalized hesitant fuzzy power averaging (GHFPA) operator, weighted generalized hesitant fuzzy 
power averaging (WGHFPA) operator, hesitant fuzzy power ordered weighted averaging (HFPOWA) operator, and generalized hesitant fuzzy power ordered weighted averaging (GHFPOWA) operator. Lin et al. [14] proposed hesitant fuzzy linguistic set (HFLS) and developed some hesitant fuzzy linguistic aggregation operators. In addition to the aforementioned aggregation operators for hesitant fuzzy information, many other research topics have also been discussed with the help of HFSs [15-26].

Recently, Zhu et al. [27] proposed dual hesitant fuzzy sets (DHFSs), which consists of two parts: the membership hesitancy function and the nonmembership hesitancy function. They have investigated some basic operations and properties of DHFS. Furthermore, Wang et al. [28] developed some aggregation operators based on dual hesitant fuzzy elements (DHFEs), such as the dual hesitant fuzzy weighted averaging (DHFWA) operator, the dual hesitant fuzzy weighted geometric (DHFWG) operator, the dual hesitant fuzzy ordered weighted averaging (DHFOWA) operator, the dual hesitant fuzzy ordered weighted geometric (DHFOWG) operator, the dual hesitant fuzzy hybrid averaging (DHFHA) operator, and the dual hesitant fuzzy hybrid geometric (DHFHG) operator, and then studied some properties of these operators. Ju et al. [29] developed some aggregation operators with intervalvalued dual hesitant fuzzy information.

It is obvious that the aforementioned aggregation operators all built on the basic algebraic product and algebraic sum, which are not the unique operations that can be chosen to model the intersection and union of IVDHFEs. Einstein operations include Einstein product and Einstein sum, which are good alternatives to the algebraic product and algebraic sum, respectively. Moreover, it seems that there are some investigations on aggregation techniques using the Einstein operations on IFSs (or HFSs) for aggregating a collection of IFVs (or HFEs). Zhao and Wei [30] applied the intuitionistic fuzzy Einstein hybrid averaging operator and intuitionistic fuzzy Einstein hybrid geometric operator to deal with MADM problems. Wang and Liu [31, 32] developed some arithmetic aggregation operators and geometric aggregation operators by using Einstein operations to aggregate intuitionistic fuzzy information. Wang and Liu $[33,34]$ further investigated the Einstein operators under interval-valued intuitionistic fuzzy environments. Zhang and Yu [35] proposed some geometric Choquet aggregation operators using Einstein operations to deal with MADM problems. Zhao et al. [36] utilized Einstein operations to develop some hesitant fuzzy correlated aggregation operators. Wei and Zhao [37] developed some induced hesitant interval-valued fuzzy Einstein aggregation operators to deal with MADM problems with hesitant interval-valued fuzzy information. Zhao et al. [38] developed some hesitant triangular fuzzy aggregation operators based on the Einstein operations.

Based on the above analysis, we find that how to extend the Einstein operations to aggregate the interval-valued dual hesitation fuzzy information is a meaningful work. Therefore, we will develop some aggregation operators based on Einstein operations under interval-valued dual hesitant fuzzy setting. To do that, the remainder of the paper is organized as follows: Section 2 reviews some basic concepts related to HFSs, DHFSs, and IVDHFSs. In Section 3, we define some operational laws for interval-valued dual hesitant fuzzy element (IVDHFE) based on Einstein operations and develop some aggregation operators for aggregating interval-valued dual hesitant fuzzy information based on Einstein operations of IVDHFE. Section 4 proposes a method to MADM problems under interval-valued dual hesitant fuzzy setting. A numerical example is developed to illustrate how to apply the proposed approach in Section 5, followed by concluding remarks in Section 6.

\section{Preliminaries}

In this section, we briefly review some basic notations and definitions regarding hesitant fuzzy sets, dual hesitant fuzzy sets, and interval-valued hesitant fuzzy sets.

2.1. Hesitant Fuzzy Sets. Torra and Narukawa [7] and Torra [8] firstly proposed the hesitant fuzzy set. The concept of hesitant fuzzy set (HFS) and some operation laws of hesitant fuzzy elements are given as follows.

Definition 1 (see [9]). Let $X$ be a fixed set; a hesitant fuzzy set (HFS) on $X$ is in terms of a function that when applied to $X$ returns a subset of $[0,1]$. To be easily understood, $\mathrm{Xia}$ and $\mathrm{Xu}$ [9] expressed the HFS by a mathematical symbol:

$$
E=\left\{\left\langle x, h_{E}(x)\right\rangle \mid x \in X\right\}
$$

where $h_{E}(x)$ is a set of some values in $[0,1]$, denoting the possible membership degrees of the element $x \in X$ to the set $E$. For convenience, Xia and Xu [9] called $h=h_{E}(x)$ a hesitant fuzzy element (HFE) and $H$ the set of all hesitant fuzzy elements (HFEs).

Definition 2 (see [9]). Let $h, h_{1}$, and $h_{2}$ be any three HFEs; then some operation laws about HFEs are defined as follows:

$$
\begin{aligned}
& \text { (1) } h^{\lambda}=\bigcup_{r \in h}\left\{r^{\lambda}\right\} ; \\
& \text { (2) } \lambda h=\bigcup_{r \in h}\left\{1-(1-r)^{\lambda}\right\} ; \\
& \text { (3) } h_{1} \oplus h_{2}=\bigcup_{r_{1} \in h_{1}, r_{2} \in h_{2}}\left\{r_{1}+r_{2}-r_{1} r_{2}\right\} ; \\
& \text { (4) } h_{1} \otimes h_{2}=\bigcup_{r_{1} \in h_{1}, r_{2} \in h_{2}}\left\{r_{1} r_{2}\right\} .
\end{aligned}
$$

Definition 3 (see [9]). Let $h$ be a HFE; then the score function of $h$ is determined as follows:

$$
S(h)=\frac{1}{l(h)} \sum_{r \in h} r,
$$

where $l(h)$ is the number of the elements in $h$. For two HFEs, $h_{1}$ and $h_{2}$, if $S\left(h_{1}\right)>S\left(h_{2}\right)$, then $h_{1}>h_{2} ;$ if $S\left(h_{1}\right)=S\left(h_{2}\right)$, then $h_{1}=h_{2}$. 
2.2. Dual Hesitant Fuzzy Sets. As an extension of HFS, Zhu et al. [27] developed the concept of dual hesitant fuzzy sets (DHFSs), in terms of two functions that return two sets of membership values and nonmembership values, respectively, for each element in the domain as follows.

Definition 4 (see [27]). Let $X$ be a fixed set; then a dual hesitant fuzzy set (DHFS) $E$ on $X$ is described as follows:

$$
E=\{\langle x, h(x), g(x)\rangle \mid x \in X\}
$$

in which $h(x)$ and $g(x)$ are two sets of some values in $[0,1]$, denoting the possible membership degrees and nonmembership degrees of the element $x \in X$ to the set $E$, with the conditions: $\gamma, \eta \in[0,1]$ and $0 \leq \gamma^{+}+\eta^{+} \leq 1$, where $\gamma \in h(x)$, $\eta \in g(x), \gamma^{+} \in h^{+}(x)=\bigcup_{\gamma \in h(x)} \max \{\gamma\}$, and $\eta^{+} \in g^{+}(x)=$ $\bigcup_{\eta \in g(x)} \max \{\eta\}$ for all $x \in X$. For convenience, the pair $e(x)=$ $\{h(x), g(x)\}$ is called a dual hesitant fuzzy element (DHFE) denoted by $e=\{h, g\}$.

To compare the DHESs, Zhu et al. [27] gave the following comparison laws.

Definition 5 (see [27]). Let $e_{1}=\left\{h_{1}, g_{1}\right\}$ and $e_{2}=\left\{h_{2}, g_{2}\right\}$ be any two DHFSs; then the score function of $e_{i}(i=1,2)$ is $S\left(e_{i}\right)=\left(1 / l\left(h_{i}\right)\right) \sum_{\gamma_{i} \in h_{i}} \gamma_{i}-\left(1 / l\left(g_{i}\right)\right) \sum_{\eta_{i} \in g_{i}} \eta_{i}(i=1,2)$ and the accuracy function of $e_{i}(i=1,2)$ is $P\left(e_{i}\right)=\left(1 / l\left(h_{i}\right)\right) \sum_{\gamma_{i} \in h_{i}} \gamma_{i}+$ $\left(1 / l\left(g_{i}\right)\right) \sum_{\eta_{i} \in g_{i}} \eta_{i}(i=1,2)$, where $l\left(h_{i}\right)$ and $l\left(g_{i}\right)$ are the numbers of the elements in $h_{i}$ and $g_{i}$, respectively; then consider the following:

(1) if $S\left(e_{1}\right)>S\left(e_{2}\right)$, then $e_{1}$ is superior to $e_{2}$, denoted by $e_{1}>e_{2}$

(2) if $S\left(e_{1}\right)=S\left(e_{2}\right)$, then consider the following:

(a) if $P\left(e_{1}\right)>P\left(e_{2}\right)$, then $e_{1}$ is superior to $e_{2}$, denoted by $e_{1}>e_{2}$;

(b) if $P\left(e_{1}\right)=P\left(e_{2}\right)$, then $e_{1}$ is equivalent to $e_{2}$, denoted by $e_{1}=e_{2}$.

2.3. Interval-Valued Dual Hesitant Fuzzy Set. In some real-life decision making problems, decision makers may find it hard to express their evaluation about an alternative under a specific attribute with exact and crisp values. Since the intervalvalued fuzzy set is usually more adequate or sufficient to model real-life decision problems than real numbers, Ju et al. [29] developed the interval-valued dual hesitant fuzzy set.

Definition 6 (see [29]). Let $X$ be a fixed set; then an intervalvalued dual hesitant fuzzy set (IVDHFS) $\widetilde{E}$ on $X$ is defined as

$$
\widetilde{E}=\{\langle x, \widetilde{h}(x), \widetilde{g}(x)\rangle \mid x \in X\},
$$

where $\widetilde{h}(x)$ and $\widetilde{g}(x)$ are two sets of some interval values in $[0,1]$, denoting the possible membership degrees and nonmembership degrees of the element $x \in X$ to the set $\widetilde{E}$, respectively, with the conditions: $\left[\gamma^{L}, \gamma^{U}\right],\left[\eta^{L}, \eta^{U}\right] \subset[0,1]$ and $0 \leq\left(\gamma^{U}\right)^{+}+\left(\eta^{U}\right)^{+} \leq 1$, where $\left[\gamma^{L}, \gamma^{U}\right] \in \tilde{h}(x),\left[\eta^{L}, \eta^{U}\right] \in$ $\widetilde{g}(x),\left(\gamma^{U}\right)^{+} \in \widetilde{h}^{+}(x)=\bigcup_{\left[\gamma^{L}, \gamma^{U}\right] \in \tilde{h}(x)} \max \left\{\gamma^{U}\right\}$, and $\left(\eta^{U}\right)^{+} \epsilon$ $\tilde{g}^{+}(x)=\bigcup_{\left[\eta^{L}, \eta^{U}\right] \in \tilde{g}(x)} \max \left\{\eta^{U}\right\}$ for all $x \in X$. For convenience, we call the pair $\widetilde{e}(x)=\{\widetilde{h}(x), \widetilde{g}(x)\}$ an interval-valued dual hesitant fuzzy element (IVDHFE) denoted by $\widetilde{e}=\{\tilde{h}, \widetilde{g}\}$ and $\widetilde{E}$ the set of all IVDHFEs.

Especially, if $\gamma^{L}=\gamma^{U}$ and $\eta^{L}=\eta^{U}$, then $\widetilde{E}$ reduces to a dual hesitant fuzzy set.

To compare the IVDHFEs, Ju et al. [29] give the following comparison laws.

Definition 7 (see [29]). Let $\widetilde{e}=(\widetilde{h}, \widetilde{g})=\bigcup_{\left[\gamma^{L}, \gamma^{U}\right] \in \widetilde{h},\left[\eta^{L}, \eta^{U}\right] \in \widetilde{g}^{\prime}}\left\{\left[\gamma^{L}, \gamma^{U}\right]\right.$, $\left.\left[\eta^{L}, \eta^{U}\right]\right\}$ be an interval-valued dual hesitant fuzzy element; then

$S(\widetilde{e})$

$$
=\frac{1}{2}\left(\frac{1}{l(\widetilde{h})} \sum_{\left[\gamma^{L}, \gamma^{U}\right] \in \tilde{h}}\left(\gamma^{L}+\gamma^{U}\right)-\frac{1}{l(\tilde{g})} \sum_{\left[\eta^{L}, \eta^{U}\right] \in \tilde{g}}\left(\eta^{L}+\eta^{U}\right)\right)
$$

is called the score function of $\widetilde{e}$, and

$$
\begin{aligned}
& H(\widetilde{e}) \\
& =\frac{1}{2}\left(\frac{1}{l(\widetilde{h})} \sum_{\left[\gamma^{L}, \gamma^{U}\right] \in \widetilde{h}}\left(\gamma^{L}+\gamma^{U}\right)+\frac{1}{l(\widetilde{g})} \sum_{\left[\eta^{L}, \eta^{U}\right] \in \widetilde{g}}\left(\eta^{L}+\eta^{U}\right)\right)
\end{aligned}
$$

is called the accuracy function of $\widetilde{e}$, where $l(\widetilde{h})$ and $l(\widetilde{g})$ are the numbers of interval values in $\tilde{h}$ and $\tilde{g}$, respectively.

Theorem 8 (see [29]). Let $\widetilde{e}_{1}=\left\{\widetilde{h}_{1}, \widetilde{g}_{1}\right\}$ and $\widetilde{e}_{2}=\left\{\widetilde{h}_{2}, \widetilde{g}_{2}\right\}$ be any two IVDHFEs; then one can compare them in terms of the following rules:

(1) if $S\left(\widetilde{e}_{1}\right)>S\left(\widetilde{e}_{2}\right)$, then $\widetilde{e}_{1}>\widetilde{e}_{2}$;

(2) if $S\left(\widetilde{e}_{1}\right)=S\left(\widetilde{e}_{2}\right)$, then
(a) if $H\left(\widetilde{e}_{1}\right)=H\left(\widetilde{e}_{2}\right)$, then $\widetilde{e}_{1}=\widetilde{e}_{2}$;
(b) if $H\left(\widetilde{e}_{1}\right)>H\left(\widetilde{e}_{2}\right)$, then $\widetilde{e}_{1}>\widetilde{e}_{2}$;
(c) if $H\left(\widetilde{e}_{1}\right)<H\left(\widetilde{e}_{2}\right)$, then $\widetilde{e}_{1}<\widetilde{e}_{2}$.

Based on algebraic operations of IVDHFEs, some interval-valued dual hesitant fuzzy aggregation operators can be defined as follows.

Motivated by the intuitionistic fuzzy aggregation operators developed by $\mathrm{Xu}$ [39] and Xu and Yager [40], some interval-valued dual hesitant fuzzy aggregation operators can be defined as follows based on algebraic operations of IVDHFEs.

Definition 9 (see [29]). Let $\widetilde{e}_{j}(j=1,2, \ldots, n)$ be a collection of IVDHFEs and let $\omega=\left(\omega_{1}, \omega_{2}, \ldots, \omega_{n}\right)^{T}$ be the weight vector of $\widetilde{e}_{j}(j=1,2, \ldots, n)$, with $\omega_{j} \in[0,1]$ and $\sum_{j=1}^{n} \omega_{j}=1$; then consider the following. 
(1) An interval-valued dual hesitant fuzzy weighted average (IVDHFWA) operator is defined as follows:

$\operatorname{IVDHFWA}\left(\widetilde{e}_{1}, \widetilde{e}_{2}, \ldots, \widetilde{e}_{n}\right)$

$=\bigoplus_{j=1}^{n} \omega_{j} \widetilde{e}_{j}$

$=\bigcup_{\left[\gamma_{j}^{L}, \gamma_{j}^{U}\right] \in \widetilde{h}_{j},\left[\eta_{j}^{L}, \eta_{j}^{U}\right] \in \widetilde{g}_{j}}\left\{\left\{\left[1-\prod_{j=1}^{n}\left(1-\gamma_{j}^{L}\right)^{\omega_{j}}\right.\right.\right.$,

$\left.\left.1-\prod_{j=1}^{n}\left(1-\gamma_{j}^{U}\right)^{\omega_{j}}\right]\right\}$

$\left.\left\{\left[\prod_{j=1}^{n}\left(\eta_{j}^{L}\right)^{\omega_{j}}, \prod_{i=1}^{n}\left(\eta_{j}^{U}\right)^{\omega_{j}}\right]\right\}\right\}$.

(2) An interval-valued dual hesitant fuzzy weighted geometric (IVDHFWG) operator is defined as follows:

$\operatorname{IVDHFWG}\left(\widetilde{e}_{1}, \widetilde{e}_{2}, \ldots, \widetilde{e}_{n}\right)$

$$
\begin{aligned}
&=\bigotimes_{j=1}^{n} \tilde{e}_{j}^{\omega_{j}}\left\{\left\{\left[\prod_{j=1}^{n}\left(\gamma_{j}^{L}\right)^{\omega_{j}}, \prod_{j=1}^{n}\left(\gamma_{j}^{L}\right)^{\omega_{j}}\right]\right\},\right. \\
&=\left\{\left[\begin{array}{l}
U \\
=
\end{array}\right] \widetilde{h}_{j},\left[\eta_{j}^{L}, \eta_{j}^{U}\right] \in \widetilde{g}_{j}\right. \\
&\left\{\prod_{j=1}^{n}\left(1-\eta_{j}^{L}\right)^{\omega_{j}},\right. \\
&\left.\left.\left.1-\prod_{j=1}^{n}\left(1-\eta_{j}^{U}\right)^{\omega_{j}}\right]\right\}\right\} .
\end{aligned}
$$

Definition 10 (see [29]). Let $\widetilde{e}_{j}(j=1,2, \ldots, n)$ be a collection of IVDHFEs, let $\widetilde{e}_{\sigma(j)}$ be the jth largest of them, and let $w=$ $\left(w_{1}, w_{2}, \ldots, w_{n}\right)^{T}$ be the aggregation-associated weight vector with $w_{j} \in[0,1]$ and $\sum_{j=1}^{n} w_{j}=1$; then consider the following.

(1) An interval-valued dual hesitant fuzzy ordered weighted averaging (IVDHFOWA) operator is defined as follows:

$\operatorname{IVDHFOWA}\left(\widetilde{e}_{1}, \widetilde{e}_{2}, \ldots, \widetilde{e}_{n}\right)$

$$
\begin{aligned}
& =\bigoplus_{j=1}^{n} w_{j} \tilde{e}_{\sigma(j)} \\
& =\bigcup_{\left[\gamma_{\sigma(j)}^{L}, \gamma_{\sigma(j)}^{U}\right] \in \tilde{h}_{\sigma(j)},\left[\eta_{\sigma(j)}^{L}, \eta_{\sigma(j)}^{U}\right] \in \tilde{g}_{\sigma(j)}}\left\{\left\{\left[1-\prod_{j=1}^{n}\left(1-\gamma_{\sigma(j)}^{L}\right)^{w_{j}},\right.\right.\right. \\
& \left.\left.1-\prod_{j=1}^{n}\left(1-\gamma_{\sigma(j)}^{U}\right)^{w_{j}}\right]\right\},
\end{aligned}
$$

$$
\begin{array}{r}
\left\{\left[\prod_{j=1}^{n}\left(\eta_{\sigma(j)}^{L}\right)^{w_{j}},\right.\right. \\
\left.\left.\prod_{i=1}^{n}\left(\eta_{\sigma(j)}^{U}\right)^{w_{j}}\right]\right\}
\end{array}
$$

(2) An interval-valued dual hesitant fuzzy ordered weighted geometric (IVDHFOWG) operator is defined as follows:

$$
\begin{aligned}
& \operatorname{IVDHFOWG}\left(\widetilde{e}_{1}, \widetilde{e}_{2}, \ldots, \widetilde{e}_{n}\right) \\
& =\bigotimes_{j=1}^{n}\left(\widetilde{e}_{\sigma(j)}\right)^{w_{j}} \\
& =\bigcup_{\left[\gamma_{\sigma(j)}^{L}, \gamma_{\sigma(j)}^{U}\right] \in \tilde{h}_{\sigma(j)},\left[\eta_{\sigma(j)}^{L}, \eta_{\sigma(j)}^{U}\right] \in \widetilde{g}_{\sigma(j)}}\left\{\left\{\left[\prod_{j=1}^{n}\left(\gamma_{\sigma(j)}^{L}\right)^{w_{j}},\right.\right.\right. \\
& \left.\left.\prod_{j=1}^{n}\left(\gamma_{\sigma(j)}^{U}\right)^{w_{j}}\right]\right\} \\
& \left\{\left[1-\prod_{j=1}^{n}\left(1-\eta_{\sigma(j)}^{L}\right)^{w_{j}},\right.\right. \\
& \left.\left.\left.1-\prod_{j=1}^{n}\left(1-\eta_{\sigma(j)}^{U}\right)^{w_{j}}\right]\right\}\right\} \text {. }
\end{aligned}
$$

Definition 11 (see [29]). Let $\widetilde{e}_{j}(j=1,2, \ldots, n)$ be a collection of IVDHFEs, let $\omega=\left(\omega_{1}, \omega_{2}, \ldots, \omega_{n}\right)^{T}$ be the weight vector of $\widetilde{e}_{j}(j=1,2, \ldots, n)$, with $\omega_{j} \in[0,1]$ and $\sum_{j=1}^{n} \omega_{j}=1$, and let $n$ be the balancing coefficient which plays a role of balance; then based on the location weighted vector $w=\left(w_{1}, w_{2}, \ldots, w_{n}\right)^{T}$, such that $w_{j} \in[0,1]$ and $\sum_{j=1}^{n} w_{j}=1$, some interval-valued dual hesitant fuzzy hybrid aggregation operators are defined as follows.

(1) An interval-valued dual hesitant fuzzy hybrid average (IVDHFHA) operator is defined as follows:

$$
\begin{aligned}
& \operatorname{IVDHFHA}\left(\widetilde{e}_{1}, \widetilde{e}_{2}, \ldots, \widetilde{e}_{n}\right) \\
& =\bigoplus_{j=1}^{n} w_{j} \dot{e}_{\sigma(j)} \\
& =\bigcup_{\left.\left[\dot{\gamma}_{\sigma(j)}^{L}, \dot{\gamma}_{\sigma(j)}^{U}\right] \in \dot{h}_{\sigma(j)}, \dot{\eta}_{\sigma(j)}^{L}, \dot{\eta}_{\sigma(j)}^{U}\right] \in \dot{\mathfrak{g}}_{\sigma(j)}}\left\{\left\{\left[1-\prod_{j=1}^{n}\left(1-\dot{\gamma}_{\sigma(j)}^{L}\right)^{w_{j}},\right.\right.\right. \\
& \left.\left.1-\prod_{j=1}^{n}\left(1-\dot{\gamma}_{\sigma(j)}^{U}\right)^{w_{j}}\right]\right\} \\
& \left\{\left[\prod_{j=1}^{n}\left(\dot{\eta}_{\sigma(j)}^{L}\right)^{w_{j}},\right.\right. \\
& \left.\left.\left.\prod_{j=1}^{n}\left(\dot{\eta}_{\sigma(j)}^{U}\right)^{w_{j}}\right]\right\}\right\}
\end{aligned}
$$


(2) An interval-valued dual hesitant fuzzy hybrid geometric (IVDHFHG) operator is defined as follows:

$$
\begin{aligned}
& \operatorname{IVDHFHG}\left(\widetilde{e}_{1}, \widetilde{e}_{2}, \ldots, \widetilde{e}_{n}\right) \\
& =\bigotimes_{j=1}^{n}\left(\ddot{e}_{\sigma(j)}\right)^{w_{j}} \\
& =\bigcup_{\left.\left[\ddot{\gamma}_{\sigma(j)}^{L}, \ddot{\gamma}_{\sigma(j)}^{U}\right] \in \ddot{h}_{j}, \ddot{\eta}_{\sigma(j)}^{L}, \ddot{\eta}_{\sigma(j)}^{U}\right] \in \ddot{g}_{j}}\left\{\left\{\left[\prod_{j=1}^{n}\left(\ddot{\gamma}_{\sigma(j)}^{L}\right)^{w_{j}},\right.\right.\right. \\
& \left.\left.\prod_{j=1}^{n}\left(\ddot{\gamma}_{\sigma(j)}^{U}\right)^{w_{j}}\right]\right\} \\
& \left\{\left[1-\prod_{j=1}^{n}\left(1-\ddot{\eta}_{\sigma(j)}^{L}\right)^{w_{j}},\right.\right. \\
& \left.\left.\left.1-\prod_{j=1}^{n}\left(1-\ddot{\eta}_{\sigma(j)}^{U}\right)^{w_{j}}\right]\right\}\right\}
\end{aligned}
$$

in which $\dot{e}_{\sigma(j)}$ is the $j$ th largest of interval-valued dual hesitant fuzzy weighted arguments $\dot{e}_{j}\left(\dot{e}_{j}=n \omega_{j} \widetilde{e}_{j}, j=1,2, \ldots, n\right)$, and $\ddot{e}_{\sigma(j)}$ is the $j$ th largest of interval-valued dual hesitant fuzzy weighted arguments $\ddot{e}_{j}\left(\ddot{e}_{j}=\left(\widetilde{e}_{j}\right)^{n \omega_{j}}, j=1,2, \ldots, n\right)$.

\section{Interval-Valued Dual Hesitant Fuzzy Aggregation Operators Based on Einstein Operations}

In this section, we will develop some aggregation operators to aggregate interval-valued dual hesitant fuzzy information based on Einstein operations. Einstein operations include the Einstein product and Einstein sum. Einstein product $\otimes_{\varepsilon}$ is a $t$-norm and Einstein sum $\oplus_{\varepsilon}$ is a $t$-conorm [41], where

(1) $a \oplus_{\varepsilon} b=(a+b) /(1+a \times b)$;

(2) $a \otimes_{\varepsilon} b=(a \times b) /(1+(1-a) \times(1-b)), \forall(a, b) \in[0,1]^{2}$.

3.1. Operational Laws for IVDHFEs Based on Einstein Operations. Let $\tilde{e}=(\tilde{h}, \widetilde{g}), \tilde{e}_{1}=\left(\tilde{h}_{1}, \tilde{g}_{1}\right)$, and $\tilde{e}_{2}=\left(\tilde{h}_{2}, \tilde{g}_{2}\right)$ be any three interval-valued dual hesitant fuzzy elements, where $\left[\gamma_{i}^{L}, \gamma_{i}^{U}\right] \in \tilde{h}_{i},\left[\eta_{i}^{L}, \eta_{i}^{U}\right] \in \tilde{g}_{i}, i=1,2$, and $\lambda>0$; then some operational laws of the IVDHFEs can be defined based on Einstein operations.

Definition 12. Let $\widetilde{e}, \widetilde{e}_{1}$, and $\widetilde{e}_{2}$ be three IVDHFEs, then we have the following operational rules:

(1) $\widetilde{e}_{1} \oplus \widetilde{e}_{2}=\bigcup_{\left.\left[\gamma_{i}^{L}, \gamma_{i}^{U}\right] \in \widetilde{h}_{i}, \eta_{i}^{L}, \eta_{i}^{U}\right] \in \widetilde{g}_{i}}\left\{\left\{\left[\frac{\gamma_{1}^{L}+\gamma_{2}^{L}}{1+\gamma_{1}^{L} \gamma_{2}^{L}}, \frac{\gamma_{1}^{U}+\gamma_{2}^{U}}{1+\gamma_{1}^{U} \gamma_{2}^{U}}\right]\right\}\right.$,

$$
\left.\left\{\left[\frac{\eta_{1}^{L} \eta_{2}^{L}}{1+\left(1-\eta_{1}^{L}\right)\left(1-\eta_{2}^{L}\right)}, \frac{\eta_{1}^{U} \eta_{2}^{U}}{1+\left(1-\eta_{1}^{U}\right)\left(1-\eta_{2}^{U}\right)}\right]\right\}\right\}, \quad i=1,2
$$

(2) $\widetilde{e}_{1} \otimes \widetilde{e}_{2}=\bigcup_{\left.\left[\gamma_{i}^{L}, \gamma_{i}^{U}\right] \in \widetilde{h}_{i}, \eta_{\eta_{i}}^{L}, \eta_{i}^{U}\right] \in \tilde{g}_{i}}\left\{\left\{\left[\frac{\gamma_{1}^{L} \gamma_{2}^{L}}{1+\left(1-\gamma_{1}^{L}\right)\left(1-\gamma_{2}^{L}\right)}, \frac{\gamma_{1}^{U} \gamma_{2}^{U}}{1+\left(1-\gamma_{1}^{U}\right)\left(1-\gamma_{2}^{U}\right)}\right]\right\}\right.$,

$$
\left.\left\{\left[\frac{\eta_{1}^{L}+\eta_{2}^{L}}{1+\eta_{1}^{L} \eta_{2}^{L}}, \frac{\eta_{1}^{U}+\eta_{2}^{U}}{1+\eta_{1}^{U} \eta_{2}^{U}}\right]\right\}\right\}, \quad i=1,2
$$

(3) $\lambda \widetilde{e}=\bigcup_{\left[\gamma^{L}, \gamma^{U}\right] \in \widetilde{h},\left[\eta^{L}, \eta^{U}\right] \in \widetilde{g}}\left\{\left\{\left[\frac{\left(1+\gamma^{L}\right)^{\lambda}-\left(1-\gamma^{L}\right)^{\lambda}}{\left(1+\gamma^{L}\right)^{\lambda}+\left(1-\gamma^{L}\right)^{\lambda}}, \frac{\left(1+\gamma^{U}\right)^{\lambda}-\left(1-\gamma^{U}\right)^{\lambda}}{\left(1+\gamma^{U}\right)^{\lambda}+\left(1-\gamma^{U}\right)^{\lambda}}\right]\right\}\right.$,

$$
\left.\left\{\left[\frac{2\left(\eta^{L}\right)^{\lambda}}{\left(2-\eta^{L}\right)^{\lambda}+\left(\eta^{L}\right)^{\lambda}}, \frac{2\left(\eta^{U}\right)^{\lambda}}{\left(2-\eta^{U}\right)^{\lambda}+\left(\eta^{U}\right)^{\lambda}}\right]\right\}\right\}, \quad \lambda>0 ;
$$

(4) $\widetilde{e}^{\lambda}=\bigcup_{\left[\gamma^{L}, \gamma^{U}\right] \in \widetilde{h},\left[\eta^{L}, \eta^{U}\right] \in \tilde{g}}\left\{\left\{\left[\frac{2\left(\gamma^{L}\right)^{\lambda}}{\left(2-\gamma^{L}\right)^{\lambda}+\left(\gamma^{L}\right)^{\lambda}}, \frac{2\left(\gamma^{U}\right)^{\lambda}}{\left(2-\gamma^{U}\right)^{\lambda}+\left(\gamma^{U}\right)^{\lambda}}\right]\right\}\right.$,

$$
\left.\left\{\left[\frac{\left(1+\eta^{L}\right)^{\lambda}-\left(1-\eta^{L}\right)^{\lambda}}{\left(1+\eta^{L}\right)^{\lambda}+\left(1-\eta^{L}\right)^{\lambda}}, \frac{\left(1+\eta^{U}\right)^{\lambda}-\left(1-\eta^{U}\right)^{\lambda}}{\left(1+\eta^{U}\right)^{\lambda}+\left(1-\eta^{U}\right)^{\lambda}}\right]\right\}\right\}, \quad \lambda>0 .
$$


Obviously, the above operational rules are still IVDHFEs. Some relationships can be further established for these operations on IVDHFEs.

Theorem 13. Let $\widetilde{e}, \tilde{e}_{1}$, and $\widetilde{e}_{2}$ be any three IVDHFEs; then one has

(1) $\widetilde{e}_{1} \oplus \widetilde{e}_{2}=\widetilde{e}_{2} \oplus \widetilde{e}_{1}$;

(2) $\tilde{e}_{1} \otimes \tilde{e}_{2}=\tilde{e}_{2} \otimes \tilde{e}_{1}$;

(3) $\lambda\left(\widetilde{e}_{1} \oplus \widetilde{e}_{2}\right)=\lambda \widetilde{e}_{1} \oplus \lambda \widetilde{e}_{2}, \lambda>0$;

(4) $\left(\widetilde{e}_{1} \otimes \widetilde{e}_{2}\right)^{\lambda}=\widetilde{e}_{1}^{\lambda} \otimes \widetilde{e}_{2}^{\lambda}, \lambda>0$.

3.2. Interval-Valued Dual Hesitant Fuzzy Einstein Weighted Aggregation Operators. Based on the above operational laws, we develop a new operator, which is defined as follows.
Definition 14. Let $\widetilde{e}_{j}(j=1,2, \ldots, n)$ be a collection of IVDHFEs; an interval-valued dual hesitant fuzzy Einstein weighted averaging (IVDHFEWA) operator is a mapping IVDHFEWA: $\widetilde{E}^{n} \rightarrow \widetilde{E}$, such that

$$
\operatorname{IVDHFEWA}\left(\widetilde{e}_{1}, \tilde{e}_{2}, \ldots, \widetilde{e}_{n}\right)=\bigoplus_{j=1}^{n}\left(\omega_{j} \tilde{e}_{j}\right)
$$

where $\omega=\left(\omega_{1}, \omega_{2}, \ldots, \omega_{n}\right)^{T}$ is the weight vector of $\left(\widetilde{e}_{1}, \widetilde{e}_{2}\right.$, $\left.\ldots, \widetilde{e}_{n}\right)$ with $\omega_{j} \in[0,1]$ and $\sum_{j=1}^{n} \omega_{j}=1$.

Theorem 15. Let $\widetilde{e}_{j}(j=1,2, \ldots, n)$ be a collection of IVDHFEs; then their aggregation value by using the IVDHFEWA operator is also an IVDHFE, and

$$
\begin{aligned}
& \operatorname{IVDHFEWA}\left(\widetilde{e}_{1}, \widetilde{e}_{2}, \ldots, \widetilde{e}_{n}\right) \\
& =\bigoplus_{j=1}^{n} w_{j} \tilde{e}_{j} \\
& =\underset{\left[\gamma_{j}^{L}, \gamma_{j}^{U}\right] \in \widetilde{h}_{j},\left[\eta_{j}^{L}, \eta_{j}^{U}\right] \in \widetilde{g}_{j}}{\bigcup_{j=1}}\left\{\left\{\frac{\prod_{j=1}^{n}\left(1+\gamma_{j}^{L}\right)^{\omega_{j}}-\prod_{j=1}^{n}\left(1-\gamma_{j}^{L}\right)^{\omega_{j}}}{\prod_{j=1}^{n}\left(1+\gamma_{j}^{L}\right)^{\omega_{j}}+\prod_{j=1}^{n}\left(1-\gamma_{j}^{L}\right)^{\omega_{j}}}, \frac{\prod_{j=1}^{n}\left(1+\gamma_{j}^{U}\right)^{\omega_{j}}-\prod_{j=1}^{n}\left(1-\gamma_{j}^{U}\right)^{\omega_{j}}}{\prod_{j=1}^{n}\left(1+\gamma_{j}^{U}\right)^{\omega_{j}}+\prod_{j=1}^{n}\left(1-\gamma_{j}^{U}\right)^{\omega_{j}}}\right]\right\}, \\
& \left.\left\{\left[\frac{2 \prod_{j=1}^{n}\left(\eta_{j}^{L}\right)^{\omega_{j}}}{\prod_{j=1}^{n}\left(2-\eta_{j}^{L}\right)^{\omega_{j}}+\prod_{j=1}^{n}\left(\eta_{j}^{L}\right)^{\omega_{j}}}, \frac{2 \prod_{j=1}^{n}\left(\eta_{j}^{U}\right)^{\omega_{j}}}{\prod_{j=1}^{n}\left(2-\eta_{j}^{U}\right)^{\omega_{j}}+\prod_{j=1}^{n}\left(\eta_{j}^{U}\right)^{\omega_{j}}}\right]\right\}\right\},
\end{aligned}
$$

where $\omega=\left(\omega_{1}, \omega_{2}, \ldots, \omega_{n}\right)^{T}$ is the weight vector of $\left(\widetilde{e}_{1}, \widetilde{e}_{2}\right.$, $\left.\ldots, \widetilde{e}_{n}\right)$ with $\omega_{j} \in[0,1]$ and $\sum_{j=1}^{n} \omega_{j}=1$.

Proof. The first result follows quickly from Definition 14. In what follows, we prove (16) using mathematical induction on $n$.

(1) When $n=1$, it is easy to conclude that (16) holds according to the Einstein operational law (3) in Definition 12:

\section{$\operatorname{IVDHFEWA}\left(\widetilde{e}_{1}\right)$}

$=w_{1} \widetilde{e}_{1}$

$$
=\bigcup_{\left[\gamma_{1}^{L}, \gamma_{1}^{U}\right] \in h_{1},\left[\eta_{1}^{L}, \eta_{1}^{U}\right] \in g_{1}}\left\{\left\{\left[\frac{\left(1+\gamma_{1}^{L}\right)^{w_{1}}-\left(1-\gamma_{1}^{L}\right)^{w_{1}}}{\left(1+\gamma_{1}^{L}\right)^{w_{1}}+\left(1-\gamma_{1}^{L}\right)^{w_{1}}},\right.\right.\right.
$$

$$
\left.\left.\frac{\left(1+\gamma_{1}^{U}\right)^{w_{1}}-\left(1-\gamma_{1}^{U}\right)^{w_{1}}}{\left(1+\gamma_{1}^{U}\right)^{w_{1}}+\left(1-\gamma_{1}^{U}\right)^{w_{1}}}\right]\right\}
$$

$$
\left\{\left[\frac{2\left(\eta_{1}^{L}\right)^{w_{1}}}{\left(2-\eta_{1}^{L}\right)^{w_{1}}+\left(\eta_{1}^{L}\right)^{w_{1}}},\right.\right.
$$

$$
\left.\left.\left.\frac{2\left(\eta_{1}^{U}\right)^{w_{1}}}{\left(2-\eta_{1}^{U}\right)^{w_{1}}+\left(\eta_{1}^{U}\right)^{w_{1}}}\right]\right\}\right\}
$$


(2) Assume that (16) holds for $n=k(k \geq 1)$; namely,

$\operatorname{IVDHFEWA}\left(\widetilde{e}_{1}, \widetilde{e}_{2}, \ldots, \widetilde{e}_{k}\right)$

$$
\begin{aligned}
=\bigoplus_{j=1}^{k} w_{j} \tilde{e}_{j} \\
=\bigcup_{\left[\gamma_{j}^{L}, \gamma_{j}^{U}\right] \in h_{j},\left[\eta_{j}^{L}, \eta_{j}^{U}\right] \in g_{j}}\{\{ \\
\left.\left\{\frac{\prod_{j=1}^{k}\left(1+\gamma_{j}^{L}\right)^{w_{j}}-\prod_{j=1}^{k}\left(1-\gamma_{j}^{L}\right)^{w_{j}}}{\prod_{j=1}^{k}\left(1+\gamma_{j}^{L}\right)^{w_{j}}+\prod_{j=1}^{k}\left(1-\gamma_{j}^{L}\right)^{w_{j}}}, \frac{\prod_{j=1}^{k}\left(1+\gamma_{j}^{U}\right)^{w_{j}}-\prod_{j=1}^{k}\left(1-\gamma_{j}^{U}\right)^{w_{j}}}{\prod_{j=1}^{k}\left(1+\gamma_{j}^{U}\right)^{w_{j}}+\prod_{j=1}^{k}\left(1-\gamma_{j}^{U}\right)^{w_{j}}}\right]\right\}, \\
\left.\left\{\left[\frac{2 \prod_{j=1}^{k}\left(\eta_{j}^{L}\right)^{w_{j}}}{\prod_{j=1}^{k}\left(2-\eta_{j}^{L}\right)^{w_{j}}+\prod_{j=1}^{k}\left(\eta_{j}^{L}\right)^{w_{j}},} \frac{2 \prod_{j=1}^{k}\left(\eta_{j}^{U}\right)^{w_{j}}}{\prod_{j=1}^{k}\left(2-\eta_{j}^{U}\right)^{w_{j}}+\prod_{j=1}^{k}\left(\eta_{j}^{U}\right)^{w_{j}}}\right]\right\}\right\} .
\end{aligned}
$$

When $n=k+1$, we get

$\operatorname{IVDHFEWA}\left(\widetilde{e}_{1}, \widetilde{e}_{2}, \ldots, \widetilde{e}_{k+1}\right)$

$$
\begin{aligned}
& =\bigoplus_{j=1}^{k+1} w_{j} \tilde{e}_{j}=\left(\bigoplus_{j=1}^{k} w_{j} \tilde{e}_{j}\right) \oplus w_{k+1} \tilde{e}_{k+1} \\
& =\operatorname{IVDHFEWA}\left(\widetilde{e}_{1}, \widetilde{e}_{2}, \ldots, \widetilde{e}_{k}\right) \oplus w_{k+1} \widetilde{e}_{k+1} \\
& =\bigcup_{\left[\gamma_{j}^{L}, \gamma_{j}^{U}\right] \in h_{j},\left[\eta_{j}^{L}, \eta_{j}^{U}\right] \in g_{j}}\left\{\left\{\left[\frac{\prod_{j=1}^{k}\left(1+\gamma_{j}^{L}\right)^{w_{j}}-\prod_{j=1}^{k}\left(1-\gamma_{j}^{L}\right)^{w_{j}}}{\prod_{j=1}^{k}\left(1+\gamma_{j}^{L}\right)^{w_{j}}+\prod_{j=1}^{k}\left(1-\gamma_{j}^{L}\right)^{w_{j}}}, \frac{\prod_{j=1}^{k}\left(1+\gamma_{j}^{U}\right)^{w_{j}}-\prod_{j=1}^{k}\left(1-\gamma_{j}^{U}\right)^{w_{j}}}{\prod_{j=1}^{k}\left(1+\gamma_{j}^{U}\right)^{w_{j}}+\prod_{j=1}^{k}\left(1-\gamma_{j}^{U}\right)^{w_{j}}}\right]\right\},\right. \\
& \left.\left\{\left[\frac{2 \prod_{j=1}^{k}\left(\eta_{j}^{L}\right)^{w_{j}}}{\prod_{j=1}^{k}\left(2-\eta_{j}^{L}\right)^{w_{j}}+\prod_{j=1}^{k}\left(\eta_{j}^{L}\right)^{w_{j}}}, \frac{2 \prod_{j=1}^{k}\left(\eta_{j}^{U}\right)^{w_{j}}}{\prod_{j=1}^{k}\left(2-\eta_{j}^{U}\right)^{w_{j}}+\prod_{j=1}^{k}\left(\eta_{j}^{U}\right)^{w_{j}}}\right]\right\}\right\} \\
& \oplus \bigcup_{\left[\gamma_{k+1}^{L}, \gamma_{k+1}^{U}\right] \in h_{k+1},\left[\eta_{k+1}^{L}, \eta_{k+1}^{U}\right] \in g_{k+1}}\left\{\left\{\left[\frac{\left(1+\gamma_{k+1}^{L}\right)^{w_{k+1}}-\left(1-\gamma_{k+1}^{L}\right)^{w_{k+1}}}{\left(1+\gamma_{k+1}^{L}\right)^{w_{1}}+\left(1-\gamma_{k+1}^{L}\right)^{w_{k+1}}}, \frac{\left(1+\gamma_{k+1}^{U}\right)^{w_{k+1}}-\left(1-\gamma_{k+1}^{U}\right)^{w_{k+1}}}{\left(1+\gamma_{k+1}^{U}\right)^{w_{k+1}}+\left(1-\gamma_{k+1}^{U}\right)^{w_{k+1}}}\right]\right\},\right. \\
& \left.\left\{\left[\frac{2\left(\eta_{k+1}^{L}\right)^{w_{k+1}}}{\left(2-\eta_{k+1}^{L}\right)^{w_{k+1}}+\left(\eta_{k+1}^{L}\right)^{w_{k+1}}}, \frac{2\left(\eta_{k+1}^{U}\right)^{w_{k+1}}}{\left(2-\eta_{k+1}^{U}\right)^{w_{k+1}}+\left(\eta_{k+1}^{U}\right)^{w_{k+1}}}\right]\right\}\right\} .
\end{aligned}
$$

Let

$$
\begin{array}{cl}
a_{1}=\prod_{j=1}^{k}\left(1+\gamma_{j}^{L}\right)^{w_{j}}, & b_{1}=\prod_{j=1}^{k}\left(1-\gamma_{j}^{L}\right)^{w_{j}}, \\
c_{1}=\prod_{j=1}^{k}\left(1+\gamma_{j}^{U}\right)^{w_{j}}, & d_{1}=\prod_{j=1}^{k}\left(1-\gamma_{j}^{U}\right)^{w_{j}}, \\
u_{1}=\prod_{j=1}^{k}\left(\eta_{j}^{L}\right)^{w_{j}}, & v_{1}=\prod_{j=1}^{k}\left(2-\eta_{j}^{L}\right)^{w_{j}},
\end{array}
$$

$$
\begin{array}{cl}
p_{1}=\prod_{j=1}^{k}\left(\eta_{j}^{U}\right)^{w_{j}}, & q_{1}=\prod_{j=1}^{k}\left(2-\eta_{j}^{U}\right)^{w_{j}}, \\
a_{2}=\left(1+\gamma_{k+1}^{L}\right)^{w_{k+1}}, & b_{2}=\left(1-\gamma_{k+1}^{L}\right)^{w_{k+1}}, \\
c_{2}=\left(1+\gamma_{k+1}^{U}\right)^{w_{k+1}}, & d_{2}=\left(1-\gamma_{k+1}^{U}\right)^{w_{k+1}}, \\
u_{2}=\left(\eta_{k+1}^{L}\right)^{w_{k+1}}, & v_{2}=\left(2-\eta_{k+1}^{L}\right)^{w_{k+1}}, \\
p_{2}=\left(\eta_{k+1}^{U}\right)^{w_{k+1}}, & q_{2}=\left(2-\eta_{k+1}^{U}\right)^{w_{k+1}} ;
\end{array}
$$


then

$\operatorname{IVDHFEWA~}\left(\widetilde{e}_{1}, \widetilde{e}_{2}, \ldots, \widetilde{e}_{k}\right)$

$=\bigcup_{\left.\left[\gamma_{j}^{L}, \gamma_{j}^{U}\right] \in h_{j}, \eta_{j}^{L}, \eta_{j}^{U}\right] \in g_{j}}\left\{\left\{\left[\frac{a_{1}-b_{1}}{a_{1}+b_{1}}, \frac{c_{1}-d_{1}}{c_{1}+d_{1}}\right]\right\}\right.$,

$\left.\left\{\left[\frac{2 u_{1}}{u_{1}+v_{1}}, \frac{2 p_{1}}{p_{1}+q_{1}}\right]\right\}\right\}$ $w_{k+1} \widetilde{e}_{k+1}$

$$
\begin{aligned}
= & \bigcup_{\left[\gamma_{k+1}^{L}, \gamma_{k+1}^{U}\right] \in h_{k+1},\left[\eta_{k+1}^{L}, \eta_{k+1}^{U}\right] \in g_{k+1}}\left\{\left\{\left[\frac{a_{2}-b_{2}}{a_{2}+b_{2}}, \frac{c_{2}-d_{2}}{c_{2}+d_{2}}\right]\right\},\right. \\
& \left.\left\{\left[\frac{2 u_{2}}{u_{2}+v_{2}}, \frac{2 p_{2}}{p_{2}+q_{2}}\right]\right\}\right\} .
\end{aligned}
$$

According to the Einstein operational law (1) in Definition 12, we have

$$
\begin{aligned}
& \operatorname{IVDHFEWA~}\left(\widetilde{e}_{1}, \widetilde{e}_{2}, \ldots, \widetilde{e}_{k+1}\right) \\
& =\bigcup_{\left.\left[\gamma_{j}^{L}, \gamma_{j}^{U}\right] \in h_{j}, \eta_{j}^{L}, \eta_{j}^{U}\right] \in g_{j}}\left\{\left\{\left[\frac{a_{1}-b_{1}}{a_{1}+b_{1}}, \frac{c_{1}-d_{1}}{c_{1}+d_{1}}\right]\right\},\left\{\left[\frac{2 u_{1}}{u_{1}+v_{1}}, \frac{2 p_{1}}{p_{1}+q_{1}}\right]\right\}\right\} \\
& \oplus \bigcup_{\left.\left[\gamma_{k+1}^{L}, \gamma_{k+1}^{U}\right] \in h_{k+1}, \eta_{k+1}^{L}, \eta_{k+1}^{U}\right] \in g_{k+1}}\left\{\left\{\left[\frac{a_{2}-b_{2}}{a_{2}+b_{2}}, \frac{c_{2}-d_{2}}{c_{2}+d_{2}}\right]\right\},\left\{\left[\frac{2 u_{2}}{u_{2}+v_{2}}, \frac{2 p_{2}}{p_{2}+q_{2}}\right]\right\}\right\} \\
& =\bigcup_{\left.\left[\gamma_{j}^{L}, \gamma_{j}^{U}\right] \in h_{j}, \eta_{j}^{L}, \eta_{j}^{U}\right] \in g_{j}}\left\{\left\{\left[\frac{a_{1} a_{2}-b_{1} b_{2}}{a_{1} a_{2}+b_{1} b_{2}}, \frac{c_{1} c_{2}-d_{1} d_{2}}{c_{1} c_{2}+d_{1} d_{2}}\right]\right\},\left\{\left[\frac{2 u_{1} u_{2}}{u_{1} u_{2}+v_{1} v_{2}}, \frac{2 p_{1} p_{2}}{p_{1} p_{2}+q_{1} q_{2}}\right]\right\}\right\} \\
& =\bigcup_{\left[\gamma_{j}^{L}, \gamma_{j}^{U}\right] \in h_{j},\left[\eta_{j}^{L}, \eta_{j}^{U}\right] \in g_{j}}\left\{\left\{\left[\frac{\prod_{j=1}^{k+1}\left(1+\gamma_{j}^{L}\right)^{w_{j}}-\prod_{j=1}^{k}\left(1-\gamma_{j}^{L}\right)^{w_{j}}}{\prod_{j=1}^{k+1}\left(1+\gamma_{j}^{L}\right)^{w_{j}}+\prod_{j=1}^{k}\left(1-\gamma_{j}^{L}\right)^{w_{j}}}, \frac{\prod_{j=1}^{k+1}\left(1+\gamma_{j}^{U}\right)^{w_{j}}-\prod_{j=1}^{k+1}\left(1-\gamma_{j}^{U}\right)^{w_{j}}}{\prod_{j=1}^{k+1}\left(1+\gamma_{j}^{U}\right)^{w_{j}}+\prod_{j=1}^{k+1}\left(1-\gamma_{j}^{U}\right)^{w_{j}}}\right]\right\},\right. \\
& \left.\left\{\left[\frac{\prod_{j=1}^{k+1}\left(1+\gamma_{j}^{L}\right)^{w_{j}}-\prod_{j=1}^{k}\left(1-\gamma_{j}^{L}\right)^{w_{j}}}{\prod_{j=1}^{k+1}\left(1+\gamma_{j}^{L}\right)^{w_{j}}+\prod_{j=1}^{k}\left(1-\gamma_{j}^{L}\right)^{w_{j}}}, \frac{\prod_{j=1}^{k+1}\left(1+\gamma_{j}^{U}\right)^{w_{j}}-\prod_{j=1}^{k+1}\left(1-\gamma_{j}^{U}\right)^{w_{j}}}{\prod_{j=1}^{k+1}\left(1+\gamma_{j}^{U}\right)^{w_{j}}+\prod_{j=1}^{k+1}\left(1-\gamma_{j}^{U}\right)^{w_{j}}}\right]\right\}\right\} ;
\end{aligned}
$$

that is, (16) holds for $n=k+1$.

According to steps (1) and (2), we know that (16) holds for any positive integer $n$.

The proof is completed.
Especially, if $\omega=(1 / n, 1 / n, \ldots, 1 / n)^{T}$, then the IVDHFEWA operator is reduced to an interval-valued dual hesitant fuzzy Einstein averaging (IVDHFEA) operator, which is shown as follows:

$\operatorname{IVDHFEA}\left(\widetilde{e}_{1}, \widetilde{e}_{2}, \ldots, \widetilde{e}_{n}\right)$

$$
\begin{aligned}
=\frac{1}{n} \bigoplus_{j=1}^{n} \widetilde{e}_{j} & =\bigcup_{\left[\gamma_{j}^{L}, \gamma_{j}^{U}\right] \in \widetilde{h}_{j},\left[\eta_{j}^{L}, \eta_{j}^{U}\right] \in \widetilde{g}_{j}}\left\{\left\{\left[\frac{\prod_{j=1}^{n}\left(1+\gamma_{j}^{L}\right)^{1 / n}-\prod_{j=1}^{n}\left(1-\gamma_{j}^{L}\right)^{1 / n}}{\prod_{j=1}^{n}\left(1+\gamma_{j}^{L}\right)^{1 / n}+\prod_{j=1}^{n}\left(1-\gamma_{j}^{L}\right)^{1 / n}}, \frac{\prod_{j=1}^{n}\left(1+\gamma_{j}^{U}\right)^{1 / n}-\prod_{j=1}^{n}\left(1-\gamma_{j}^{U}\right)^{1 / n}}{\prod_{j=1}^{n}\left(1+\gamma_{j}^{U}\right)^{1 / n}+\prod_{j=1}^{n}\left(1-\gamma_{j}^{U}\right)^{1 / n}}\right]\right\},\right. \\
& \left.\left\{\left[\frac{2 \prod_{j=1}^{n}\left(\eta_{j}^{L}\right)^{1 / n}}{\prod_{j=1}^{n}\left(2-\eta_{j}^{L}\right)^{1 / n}+\prod_{j=1}^{n}\left(\eta_{j}^{L}\right)^{1 / n}}, \frac{2 \prod_{j=1}^{n}\left(\eta_{j}^{U}\right)^{1 / n}}{\prod_{j=1}^{n}\left(2-\eta_{j}^{U}\right)^{1 / n}+\prod_{j=1}^{n}\left(\eta_{j}^{U}\right)^{1 / n}}\right]\right\}\right\} .
\end{aligned}
$$


Theorem 16. Let $\widetilde{e}_{j}(j=1,2, \ldots, n)$ be a collection of IVDHFEs, then we have the following properties:

(1) Idempotency. If all $\widetilde{e}_{j}(j=1,2, \ldots, n)$ are equal and $\widetilde{e}_{j}$ $=\widetilde{e}=\left\{\left\{\left[\gamma^{L}, \gamma^{U}\right]\right\},\left\{\left[\eta^{L}, \eta^{U}\right]\right\}\right\}$, for all $j=1,2, \ldots, n$, then

$$
\operatorname{IVDHFEWA}\left(\widetilde{e}_{1}, \tilde{e}_{2}, \ldots, \widetilde{e}_{n}\right)=\tilde{e} .
$$

Proof. Since $\widetilde{e}_{j}=\widetilde{e}=\left\{\left\{\left[\gamma^{L}, \gamma^{U}\right]\right\},\left\{\left[\eta^{L}, \eta^{U}\right]\right\}\right\}$, for all $j=$ $1,2, \ldots, n$, then

$$
\begin{aligned}
& \operatorname{IVDHFEWA}\left(\widetilde{e}_{1}, \widetilde{e}_{2}, \ldots, \widetilde{e}_{n}\right) \\
& =\bigcup_{\left.\left[\gamma_{j}^{L}, \gamma_{j}^{U}\right] \in h_{j}, \eta_{j}^{L}, \eta_{j}^{U}\right] \in \tilde{g}_{j}}\left\{\left\{\left[\frac{\prod_{j=1}^{n}\left(1+\gamma_{j}^{L}\right)^{\omega_{j}}-\prod_{j=1}^{n}\left(1-\gamma_{j}^{L}\right)^{\omega_{j}}}{\prod_{j=1}^{n}\left(1+\gamma_{j}^{L}\right)^{\omega_{j}}+\prod_{j=1}^{n}\left(1-\gamma_{j}^{L}\right)^{\omega_{j}}}, \frac{\prod_{j=1}^{n}\left(1+\gamma_{j}^{U}\right)^{\omega_{j}}-\prod_{j=1}^{n}\left(1-\gamma_{j}^{U}\right)^{\omega_{j}}}{\prod_{j=1}^{n}\left(1+\gamma_{j}^{U}\right)^{\omega_{j}}+\prod_{j=1}^{n}\left(1-\gamma_{j}^{U}\right)^{\omega_{j}}}\right]\right\},\right. \\
& \left\{\left[\frac{2 \prod_{j=1}^{n}\left(\eta_{j}^{L}\right)^{\omega_{j}}}{\prod_{j=1}^{n}\left(2-\eta_{j}^{L}\right)^{\omega_{j}}+\prod_{j=1}^{n}\left(\eta_{j}^{L}\right)^{\omega_{j}}}, \frac{2 \prod_{j=1}^{n}\left(\eta_{j}^{U}\right)^{\omega_{j}}}{\prod_{j=1}^{n}\left(2-\eta_{j}^{U}\right)^{\omega_{j}}+\prod_{j=1}^{n}\left(\eta_{j}^{U}\right)^{\omega_{j}}}\right]\right\} \\
& =\bigcup_{\left.\left[\gamma^{L}, \gamma^{U}\right] \in \widetilde{h}_{j}, \underline{\eta}^{L}, \eta^{U}\right] \in \tilde{g}_{j}}\left\{\left\{\left[\frac{\prod_{j=1}^{n}\left(1+\gamma^{L}\right)^{\omega_{j}}-\prod_{j=1}^{n}\left(1-\gamma^{L}\right)^{\omega_{j}}}{\prod_{j=1}^{n}\left(1+\gamma^{L}\right)^{\omega_{j}}+\prod_{j=1}^{n}\left(1-\gamma^{L}\right)^{\omega_{j}}}, \frac{\prod_{j=1}^{n}\left(1+\gamma^{U}\right)^{\omega_{j}}-\prod_{j=1}^{n}\left(1-\gamma^{U}\right)^{\omega_{j}}}{\prod_{j=1}^{n}\left(1+\gamma^{U}\right)^{\omega_{j}}+\prod_{j=1}^{n}\left(1-\gamma^{U}\right)^{\omega_{j}}}\right]\right\},\right. \\
& \left.\left\{\left[\frac{2 \prod_{j=1}^{n}\left(\eta^{L}\right)^{\omega_{j}}}{\prod_{j=1}^{n}\left(2-\eta^{L}\right)^{\omega_{j}}+\prod_{j=1}^{n}\left(\eta^{L}\right)^{\omega_{j}}}, \frac{2 \prod_{j=1}^{n}\left(\eta^{U}\right)^{\omega_{j}}}{\prod_{j=1}^{n}\left(2-\eta^{U}\right)^{\omega_{j}}+\prod_{j=1}^{n}\left(\eta^{U}\right)^{\omega_{j}}}\right]\right\}\right\} \\
& =\bigcup_{\left[\gamma^{L}, \gamma^{U}\right] \in h_{j},\left[\eta^{L}, \eta^{U}\right] \in g_{j}}\left\{\left\{\left[\frac{1+\gamma^{L}-\left(1-\gamma^{L}\right)}{1+\gamma^{L}+\left(1-\gamma^{L}\right)}, \frac{1+\gamma^{U}-\left(1-\gamma^{L}\right)}{1+\gamma^{U}+\left(1-\gamma^{L}\right)}\right]\right\},\left\{\left[\frac{2 \eta^{L}}{2-\eta^{L}+\eta^{L}}, \frac{2 \eta^{U}}{2-\eta^{U}+\eta^{U}}\right]\right\}\right\} \\
& =\bigcup_{\left[\gamma^{L}, \gamma^{U}\right] \in h_{j},\left[\eta^{L}, \eta^{U}\right] \in g_{j}}\left\{\left\{\left[\gamma^{L}, \gamma^{U}\right]\right\},\left\{\left[\eta^{L}, \eta^{U}\right]\right\}\right\} \\
& =\left\{\left\{\left[\gamma^{L}, \gamma^{U}\right]\right\},\left\{\left[\eta^{L}, \eta^{U}\right]\right\}\right\}=\widetilde{e} .
\end{aligned}
$$

Thus, $\operatorname{IVDHFEWA}\left(\widetilde{e}_{1}, \widetilde{e}_{2}, \ldots, \widetilde{e}_{n}\right)=\tilde{e}$.

The proof is completed.

(2) Boundedness. If $\gamma_{\min }^{L}=\min _{1 \leq j \leq n}\left\{\gamma_{j}^{L} \mid\left[\gamma_{j}^{L}, \gamma_{j}^{U}\right] \in \widetilde{h}_{j}\right\}$, $\gamma_{\min }^{U}=\min _{1 \leq j \leq n}\left\{\gamma_{j}^{U} \mid\left[\gamma_{j}^{L}, \gamma_{j}^{U}\right] \in \widetilde{h}_{j}\right\}, \gamma_{\max }^{L}=$ $\max _{1 \leq j \leq n}\left\{\gamma_{j}^{L} \mid\left[\gamma_{j}^{L}, \gamma_{j}^{U}\right] \in \widetilde{h}_{j}\right\}, \gamma_{\max }^{U}=\max _{1 \leq j \leq n}\left\{\gamma_{j}^{U} \mid\right.$ $\left.\left[\gamma_{j}^{L}, \gamma_{j}^{U}\right] \in \widetilde{h}_{j}\right\}, \eta_{\min }^{L}=\min _{1 \leq j \leq n}\left\{\eta_{j}^{L} \mid\left[\eta_{j}^{L}, \eta_{j}^{U}\right] \in \widetilde{g}_{j}\right\}$, $\eta_{\min }^{U}=\min _{1 \leq j \leq n}\left\{\eta_{j}^{U} \mid\left[\eta_{j}^{L}, \eta_{j}^{U}\right] \in \tilde{g}_{j}\right\}, \eta_{\max }^{L}=$ $\max _{1 \leq j \leq n}\left\{\eta_{j}^{L} \mid\left[\eta_{j}^{L}, \eta_{j}^{U}\right] \in \tilde{g}_{j}\right\}$, and $\eta_{\max }^{U}=$ $\max _{1 \leq j \leq n}\left\{\eta_{j}^{U} \mid\left[\eta_{j}^{L}, \eta_{j}^{U}\right] \in \tilde{g}_{j}\right\}$, for all $j=1,2, \ldots, n$, then we can obtain

$$
\begin{aligned}
& \left\{\left\{\left[\gamma_{\min }^{L}, \gamma_{\min }^{U}\right]\right\},\left\{\left[\eta_{\max }^{L}, \eta_{\max }^{U}\right]\right\}\right\} \\
& \quad \leq \operatorname{IVDHFEWA}\left(\widetilde{e}_{1}, \widetilde{e}_{2}, \ldots, \widetilde{e}_{n}\right) \\
& \quad \leq\left\{\left\{\left[\gamma_{\max }^{L}, \gamma_{\max }^{U}\right]\right\},\left\{\left[\eta_{\min }^{L}, \eta_{\min }^{U}\right]\right\}\right\} .
\end{aligned}
$$

Proof. Let $f(x)=(1-x) /(1+x), x \in(0,1]$; then $f^{\prime}(x)=$ $-2 /(1+x)^{2}<0$; that is, $f(x)$ is a decreasing function. Since $\gamma_{\min }^{L} \leq \gamma_{j}^{L} \leq \gamma_{\max }^{L}$, then, for all $j$, we have $f\left(\gamma_{\max }^{L}\right) \leq f\left(\gamma_{j}^{L}\right) \leq$ $f\left(\gamma_{\min }^{L}\right)$; that is,

$$
\frac{1-\gamma_{\max }^{L}}{1+\gamma_{\max }^{L}} \leq \frac{1-\gamma_{j}^{L}}{1+\gamma_{j}^{L}} \leq \frac{1-\gamma_{\min }^{L}}{1+\gamma_{\min }^{L}}
$$

Let $\omega=\left(\omega_{1}, \omega_{2}, \ldots, \omega_{n}\right)^{T}$ be the weight vector of $\left(\widetilde{e}_{1}, \widetilde{e}_{2}\right.$, $\left.\ldots, \tilde{e}_{n}\right)$, such that $\omega_{j} \in[0,1]$ and $\sum_{j=1}^{n} \omega_{j}=1$. Then, for all $\omega_{j} \in[0,1]$, we have

$$
\left(\frac{1-\gamma_{\max }^{L}}{1+\gamma_{\max }^{L}}\right)^{\omega_{j}} \leq\left(\frac{1-\gamma_{j}^{L}}{1+\gamma_{j}^{L}}\right)^{\omega_{j}} \leq\left(\frac{1-\gamma_{\min }^{L}}{1+\gamma_{\min }^{L}}\right)^{\omega_{j}}
$$


10

Mathematical Problems in Engineering

Thus

$$
\begin{aligned}
& \prod_{j=1}^{n}\left(\frac{1-\gamma_{\max }^{L}}{1+\gamma_{\max }^{L}}\right)^{\omega_{j}} \\
& \leq \prod_{j=1}^{n}\left(\frac{1-\gamma_{j}^{L}}{1+\gamma_{j}^{L}}\right)^{\omega_{j}} \leq \prod_{j=1}^{n}\left(\frac{1-\gamma_{\min }^{L}}{1+\gamma_{\min }^{L}}\right)^{\omega_{j}} \\
& \Longleftrightarrow \frac{1-\gamma_{\max }^{L}}{1+\gamma_{\max }^{L}} \leq \prod_{j=1}^{n}\left(\frac{1-\gamma_{j}^{L}}{1+\gamma_{j}^{L}}\right)^{\omega_{j}} \leq \frac{1-\gamma_{\min }^{L}}{1+\gamma_{\min }^{L}} \\
& \Longleftrightarrow \frac{2}{1+\gamma_{\max }^{L}} \leq 1+\prod_{j=1}^{n}\left(\frac{1-\gamma_{j}^{L}}{1+\gamma_{j}^{L}}\right)^{\omega_{j}} \leq \frac{2}{1+\gamma_{\min }^{L}} \\
& \Longleftrightarrow \frac{1+\gamma_{\min }^{L}}{2} \leq \frac{1}{1+\prod_{j=1}^{n}\left(\left(1-\gamma_{j}^{L}\right) /\left(1+\gamma_{j}^{L}\right)\right)^{\omega_{j}}} \\
& \leq \frac{1+\gamma_{\max }^{L}}{2} \\
& \Longleftrightarrow 1+\gamma_{\min }^{L} \leq \frac{2}{1+\prod_{j=1}^{n}\left(\left(1-\gamma_{j}^{L}\right) /\left(1+\gamma_{j}^{L}\right)\right)^{\omega_{j}}} \\
& \leq 1+\gamma_{\max }^{L} \\
& \Longleftrightarrow \gamma_{\min }^{L} \leq \frac{2}{1+\prod_{j=1}^{n}\left(\left(1-\gamma_{j}^{L}\right) /\left(1+\gamma_{j}^{L}\right)\right)^{\omega_{j}}}-1 \leq \gamma_{\max }^{L} ;
\end{aligned}
$$

that is,

$$
\gamma_{\min }^{L} \leq \frac{\prod_{j=1}^{n}\left(1+\gamma_{j}^{L}\right)^{\omega_{j}}-\prod_{j=1}^{n}\left(1-\gamma_{j}^{L}\right)^{\omega_{j}}}{\prod_{j=1}^{n}\left(1+\gamma_{j}^{L}\right)^{\omega_{j}}+\prod_{j=1}^{n}\left(1-\gamma_{j}^{L}\right)^{\omega_{j}}} \leq \gamma_{\max }^{L} .
$$

Similarly, we have

$$
\gamma_{\min }^{U} \leq \frac{\prod_{j=1}^{n}\left(1+\gamma_{j}^{U}\right)^{\omega_{j}}-\prod_{j=1}^{n}\left(1-\gamma_{j}^{U}\right)^{\omega_{j}}}{\prod_{j=1}^{n}\left(1+\gamma_{j}^{U}\right)^{\omega_{j}}+\prod_{j=1}^{n}\left(1-\gamma_{j}^{U}\right)^{\omega_{j}}} \leq \gamma_{\max }^{U} .
$$

For all $j$, we have

$$
\begin{aligned}
& \gamma_{\min }^{L}+\gamma_{\min }^{U} \\
& \leq \frac{1}{l(\widetilde{h})_{\left[\gamma^{L}, \gamma^{U}\right] \in \tilde{h}}} \sum\left(\frac{\prod_{j=1}^{n}\left(1+\gamma_{j}^{L}\right)^{\omega_{j}}-\prod_{j=1}^{n}\left(1-\gamma_{j}^{L}\right)^{\omega_{j}}}{\prod_{j=1}^{n}\left(1+\gamma_{j}^{L}\right)^{\omega_{j}}+\prod_{j=1}^{n}\left(1-\gamma_{j}^{L}\right)^{\omega_{j}}}\right. \\
& \left.+\frac{\prod_{j=1}^{n}\left(1+\gamma_{j}^{U}\right)^{\omega_{j}}-\prod_{j=1}^{n}\left(1-\gamma_{j}^{U}\right)^{\omega_{j}}}{\prod_{j=1}^{n}\left(1+\gamma_{j}^{U}\right)^{\omega_{j}}+\prod_{j=1}^{n}\left(1-\gamma_{j}^{U}\right)^{\omega_{j}}}\right) \\
& \leq \gamma_{\max }^{L}+\gamma_{\max }^{U},
\end{aligned}
$$

where $l(\widetilde{h})$ is the number of interval values in the membership degrees of IVDHFEWA $\left(\widetilde{e}_{1}, \widetilde{e}_{2}, \ldots, \widetilde{e}_{n}\right)$.

Let $g(y)=(2-y) / y, y \in(0,1]$; then $g^{\prime}(y)=-2 / y^{2}<0$; that is, $g(y)$ is a decreasing function. Since $\eta_{\min }^{L} \leq \eta_{j}^{L} \leq \eta_{\max }^{L}$, then, for all $j$, we have $g\left(\eta_{\max }^{L}\right) \leq g\left(\eta_{j}^{L}\right) \leq g\left(\eta_{\min }^{L}\right)$; that is, $(2-$ $\left.\eta_{\max }^{L}\right) / y \leq\left(2-\eta_{j}^{L}\right) / y \leq\left(2-\eta_{\min }^{L}\right) / y$. Let $\omega=\left(\omega_{1}, \omega_{2}, \ldots, \omega_{n}\right)^{T}$ be the weight vector of $\left(\widetilde{e}_{1}, \widetilde{e}_{2}, \ldots, \widetilde{e}_{n}\right)$, such that $\omega_{j} \in[0,1]$ and $\sum_{j=1}^{n} \omega_{j}=1$. Then, for all $\omega_{j} \in[0,1]$, we have

$$
\left(\frac{2-\eta_{\max }^{L}}{\eta_{\max }^{L}}\right)^{\omega_{j}} \leq\left(\frac{2-\eta_{j}^{L}}{\eta_{j}^{L}}\right)^{\omega_{j}} \leq\left(\frac{2-\eta_{\min }^{L}}{\eta_{\min }^{L}}\right)^{\omega_{j}} .
$$

Thus

$$
\begin{aligned}
\prod_{n=1}^{n}\left(\frac{2-\eta_{\max }^{L}}{\eta_{\max }^{L}}\right)^{\omega_{j}} \\
\quad \leq \prod_{n=1}^{n}\left(\frac{2-\eta_{j}^{L}}{\eta_{j}^{L}}\right)^{\omega_{j}} \leq \prod_{n=1}^{n}\left(\frac{2-\eta_{\min }^{L}}{\eta_{\min }^{L}}\right)^{\omega_{j}} \\
\Longleftrightarrow \frac{2-\eta_{\max }^{L}}{\eta_{\max }^{L}} \leq \prod_{n=1}^{n}\left(\frac{2-\eta_{j}^{L}}{\eta_{j}^{L}}\right)^{\omega_{j}} \leq \frac{2-\eta_{\min }^{L}}{\eta_{\min }^{L}} \\
\Longleftrightarrow \frac{2}{\eta_{\max }^{L}} \leq \prod_{n=1}^{n}\left(\frac{2-\eta_{j}^{L}}{\eta_{j}^{L}}\right)^{\omega_{j}}+1 \leq \frac{2}{\eta_{\min }^{L}} \\
\quad \Longleftrightarrow \frac{\eta_{\min }^{L}}{2} \leq \frac{1}{\prod_{n=1}^{n}\left(\left(2-\eta_{j}^{L}\right) / \eta_{j}^{L}\right)^{\omega_{j}}+1} \leq \frac{\eta_{\max }^{L}}{2}
\end{aligned}
$$

$$
\Longleftrightarrow \eta_{\min }^{L} \leq \frac{2}{\prod_{n=1}^{n}\left(\left(2-\eta_{j}^{L}\right) / \eta_{j}^{L}\right)^{\omega_{j}}+1} \leq \eta_{\max }^{L} ;
$$

that is,

$$
\Longleftrightarrow \eta_{\min }^{L} \leq \frac{2 \prod_{j=1}^{n}\left(\eta_{j}^{L}\right)^{\omega_{j}}}{\prod_{j=1}^{n}\left(2-\eta_{j}^{L}\right)^{\omega_{j}}+\prod_{j=1}^{n}\left(\eta_{j}^{L}\right)^{\omega_{j}}} \leq \eta_{\max }^{L} .
$$

Similarly, we have

$$
\eta_{\min }^{U} \leq \frac{2 \prod_{j=1}^{n}\left(\eta_{j}^{U}\right)^{\omega_{j}}}{\prod_{j=1}^{n}\left(2-\eta_{j}^{U}\right)^{\omega_{j}}+\prod_{j=1}^{n}\left(\eta_{j}^{U}\right)^{\omega_{j}}} \leq \eta_{\max }^{U} .
$$


That is,

$$
\begin{aligned}
& \eta_{\min }^{L}+\eta_{\min }^{U} \\
& \leq \frac{1}{l(\widetilde{g})} \sum_{\left[\eta^{L}, \eta^{U}\right] \in \tilde{g}}\left(\frac{2 \prod_{j=1}^{n}\left(\eta_{j}^{L}\right)^{\omega_{j}}}{\prod_{j=1}^{n}\left(2-\eta_{j}^{L}\right)^{\omega_{j}}+\prod_{j=1}^{n}\left(\eta_{j}^{L}\right)^{\omega_{j}}}\right. \\
& \left.+\frac{2 \prod_{j=1}^{n}\left(\eta_{j}^{U}\right)^{\omega_{j}}}{\prod_{j=1}^{n}\left(2-\eta_{j}^{U}\right)^{\omega_{j}}+\prod_{j=1}^{n}\left(\eta_{j}^{U}\right)^{\omega_{j}}}\right) \\
& \leq \eta_{\max }^{L}+\eta_{\max }^{U},
\end{aligned}
$$

where $l(\widetilde{g})$ is the number of interval values in the nonmembership degrees of IVDHFEWA $\left(\widetilde{e}_{1}, \widetilde{e}_{2}, \ldots, \widetilde{e}_{n}\right)$.

So we can get

$$
\begin{aligned}
& \frac{\left(\gamma_{\min }^{L}+\gamma_{\min }^{U}\right)-\left(\eta_{\max }^{L}+\eta_{\max }^{U}\right)}{2} \\
& \leq \frac{1}{2} \cdot \frac{1}{l(\widetilde{h})} \\
& \times \sum_{\left[\gamma^{L}, \gamma^{U}\right] \in \tilde{h}}\left(\frac{\prod_{j=1}^{n}\left(1+\gamma_{j}^{L}\right)^{\omega_{j}}-\prod_{j=1}^{n}\left(1-\gamma_{j}^{L}\right)^{\omega_{j}}}{\prod_{j=1}^{n}\left(1+\gamma_{j}^{L}\right)^{\omega_{j}}+\prod_{j=1}^{n}\left(1-\gamma_{j}^{L}\right)^{\omega_{j}}}\right. \\
& \left.\quad+\frac{\prod_{j=1}^{n}\left(1+\gamma_{j}^{U}\right)^{\omega_{j}}-\prod_{j=1}^{n}\left(1-\gamma_{j}^{U}\right)^{\omega_{j}}}{\prod_{j=1}^{n}\left(1+\gamma_{j}^{U}\right)^{\omega_{j}}+\prod_{j=1}^{n}\left(1-\gamma_{j}^{U}\right)^{\omega_{j}}}\right) \\
& \times \sum_{\left[\eta^{L}, \eta^{U}\right] \in \tilde{g}}\left(\frac{1}{\prod_{j=1}^{n}\left(2-\eta_{j}^{L}\right)^{\omega_{j}}+\prod_{j=1}^{n}\left(\eta_{j}^{L}\right)^{\omega_{j}}}\right. \\
& \leq \frac{2 \prod_{j=1}^{n}\left(\eta_{j}^{U}\right)^{\omega_{j}}}{\left.\prod_{\max }^{L}+\gamma_{\max }^{U}\right)-\left(\eta_{\min }^{L}+\eta_{\min }^{U}\right)} \\
& 2
\end{aligned}
$$

Therefore, according to Theorem 8 , we have

$$
\begin{aligned}
& \left\{\left\{\left[\gamma_{\text {min }}^{L}, \gamma_{\min }^{U}\right]\right\},\left\{\left[\eta_{\max }^{L}, \eta_{\max }^{U}\right]\right\}\right\} \\
& \quad \leq \operatorname{IVDHFEWA}\left(\widetilde{e}_{1}, \widetilde{e}_{2}, \ldots, \widetilde{e}_{n}\right) \\
& \quad \leq\left\{\left\{\left[\gamma_{\max }^{L}, \gamma_{\max }^{U}\right]\right\},\left\{\left[\eta_{\min }^{L}, \eta_{\min }^{U}\right]\right\}\right\} .
\end{aligned}
$$

The proof is completed.
Lemma 17 (see [42]). Let $a_{j}>0, w_{j}>0, j=1,2, \ldots, n$, and $\sum_{j=1}^{n} w_{j}=1$; then

$$
\prod_{j=1}^{n} a_{j}^{w_{j}} \leq \sum_{j=1}^{n} w_{j} a_{j}
$$

with equality if and only if $a_{1}=a_{2}=\cdots=a_{n}$.

To compare the aggregated values between the IVDHFEWA operator and IVDHFWA operator in (8), we give the following theorem.

Theorem 18. Let $\widetilde{e}_{j}(j=1,2, \ldots, n)$ be a collection of IVDHFEs and let $\omega=\left(\omega_{1}, \omega_{2}, \ldots, \omega_{n}\right)^{T}$ be the weight vector of $\left(\widetilde{e}_{1}, \widetilde{e}_{2}, \ldots, \widetilde{e}_{n}\right)$ with $\omega_{j} \in[0,1]$ and $\sum_{j=1}^{n} \omega_{j}=1$; then

$\operatorname{IVDHFEWA}\left(\widetilde{e}_{1}, \widetilde{e}_{2}, \ldots, \widetilde{e}_{n}\right) \leq \operatorname{IVDHFWA}\left(\widetilde{e}_{1}, \widetilde{e}_{2}, \ldots, \widetilde{e}_{n}\right)$.

Proof. According to Lemma 17, for any $\left[\gamma_{j}^{L}, \gamma_{j}^{U}\right] \in \widetilde{h}_{j}, j=$ $1,2, \ldots, n$, we have

$$
\begin{aligned}
\prod_{j=1}^{n}\left(1+\gamma_{j}^{L}\right)^{\omega_{j}}+\prod_{j=1}^{n}\left(1-\gamma_{j}^{L}\right)^{\omega_{j}} \\
\quad \leq \sum_{j=1}^{n} \omega_{j}\left(1+\gamma_{j}^{L}\right)+\sum_{j=1}^{n} \omega_{j}\left(1-\gamma_{j}^{L}\right)=2, \\
\prod_{j=1}^{n}\left(1+\gamma_{j}^{U}\right)^{\omega_{j}}+\prod_{j=1}^{n}\left(1-\gamma_{j}^{U}\right)^{\omega_{j}} \\
\quad \leq \sum_{j=1}^{n} \omega_{j}\left(1+\gamma_{j}^{U}\right)+\sum_{j=1}^{n} \omega_{j}\left(1+\gamma_{j}^{U}\right)=2 .
\end{aligned}
$$

Thus, we have

$$
\begin{aligned}
& \frac{\prod_{j=1}^{n}\left(1+\gamma_{j}^{L}\right)^{\omega_{j}}-\prod_{j=1}^{n}\left(1-\gamma_{j}^{L}\right)^{\omega_{j}}}{\prod_{j=1}^{n}\left(1+\gamma_{j}^{L}\right)^{\omega_{j}}+\prod_{j=1}^{n}\left(1-\gamma_{j}^{L}\right)^{\omega_{j}}} \\
& \quad=1-\frac{2 \prod_{j=1}^{n}\left(1-\gamma_{j}^{L}\right)^{\omega_{j}}}{\prod_{j=1}^{n}\left(1+\gamma_{j}^{L}\right)^{\omega_{j}}+\prod_{j=1}^{n}\left(1-\gamma_{j}^{L}\right)^{\omega_{j}}} \\
& \quad \leq 1-\prod_{j=1}^{n}\left(1-\gamma_{j}^{L}\right)^{\omega_{j}},
\end{aligned}
$$$$
\frac{\prod_{j=1}^{n}\left(1+\gamma_{j}^{U}\right)^{\omega_{j}}-\prod_{j=1}^{n}\left(1-\gamma_{j}^{U}\right)^{\omega_{j}}}{\prod_{j=1}^{n}\left(1+\gamma_{j}^{U}\right)^{\omega_{j}}+\prod_{j=1}^{n}\left(1-\gamma_{j}^{U}\right)^{\omega_{j}}}
$$

$$
\begin{aligned}
& =1-\frac{2 \prod_{j=1}^{n}\left(1-\gamma_{j}^{U}\right)^{\omega_{j}}}{\prod_{j=1}^{n}\left(1+\gamma_{j}^{U}\right)^{\omega_{j}}+\prod_{j=1}^{n}\left(1-\gamma_{j}^{U}\right)^{\omega_{j}}} \\
& \leq 1-\prod_{j=1}^{n}\left(1-\gamma_{j}^{U}\right)^{\omega_{j}} .
\end{aligned}
$$


Similarly, for any $\left[\eta_{j}^{L}, \eta_{j}^{U}\right] \in \widetilde{h}_{j}, j=1,2, \ldots, n$, we have

$$
\begin{gathered}
\frac{2 \prod_{j=1}^{n}\left(\eta_{j}^{L}\right)^{\omega_{j}}}{\prod_{j=1}^{n}\left(2-\eta_{j}^{L}\right)^{\omega_{j}}+\prod_{j=1}^{n}\left(\eta_{j}^{L}\right)^{\omega_{j}}} \geq \prod_{j=1}^{n}\left(\eta_{j}^{L}\right)^{\omega_{j}}, \\
\frac{2 \prod_{j=1}^{n}\left(\eta_{j}^{U}\right)^{\omega_{j}}}{\prod_{j=1}^{n}\left(2-\eta_{j}^{U}\right)^{\omega_{j}}+\prod_{j=1}^{n}\left(\eta_{j}^{U}\right)^{\omega_{j}}} \geq \prod_{j=1}^{n}\left(\eta_{j}^{U}\right)^{\omega_{j}} .
\end{gathered}
$$

That is,

$$
\begin{aligned}
& \frac{1}{l_{1}} \sum_{\left[\gamma_{j}^{L}, \gamma_{j}^{U}\right] \in \widetilde{h}_{j}}\left(\frac{\prod_{j=1}^{n}\left(1+\gamma_{j}^{L}\right)^{\omega_{j}}-\prod_{j=1}^{n}\left(1-\gamma_{j}^{L}\right)^{\omega_{j}}}{\prod_{j=1}^{n}\left(1+\gamma_{j}^{L}\right)^{\omega_{j}}+\prod_{j=1}^{n}\left(1-\gamma_{j}^{L}\right)^{\omega_{j}}}\right. \\
& \left.+\frac{\prod_{j=1}^{n}\left(1+\gamma_{j}^{U}\right)^{\omega_{j}}-\prod_{j=1}^{n}\left(1-\gamma_{j}^{U}\right)^{\omega_{j}}}{\prod_{j=1}^{n}\left(1+\gamma_{j}^{U}\right)^{\omega_{j}}+\prod_{j=1}^{n}\left(1-\gamma_{j}^{U}\right)^{\omega_{j}}}\right) \\
& \leq \frac{1}{l_{3}} \sum_{\left[\gamma_{j}^{L}, \gamma_{j}^{U}\right] \in \tilde{h}_{j}}\left(1-\prod_{j=1}^{n}\left(1-\gamma_{j}^{L}\right)^{w_{j}}+1-\prod_{j=1}^{n}\left(1-\gamma_{j}^{U}\right)^{w_{j}}\right) ; \\
& \frac{1}{l_{2}} \sum_{\left[\eta_{j}^{L}, \eta_{j}^{U}\right] \in \widetilde{g}_{j}}\left(\frac{2 \prod_{j=1}^{n}\left(\eta_{j}^{L}\right)^{\omega_{j}}}{\prod_{j=1}^{n}\left(2-\eta_{j}^{L}\right)^{\omega_{j}}+\prod_{j=1}^{n}\left(\eta_{j}^{L}\right)^{\omega_{j}}}\right. \\
& \left.+\frac{2 \prod_{j=1}^{n}\left(\eta_{j}^{U}\right)^{\omega_{j}}}{\prod_{j=1}^{n}\left(2-\eta_{j}^{U}\right)^{\omega_{j}}+\prod_{j=1}^{n}\left(\eta_{j}^{U}\right)^{\omega_{j}}}\right) \\
& \geq \frac{1}{l_{4}} \sum_{\left[\eta_{j}^{L}, \eta_{j}^{U}\right] \in \widetilde{g}_{j}}\left(\prod_{j=1}^{n}\left(\eta_{j}^{L}\right)^{\omega_{j}}+\prod_{j=1}^{n}\left(\eta_{j}^{U}\right)^{\omega_{j}}\right),
\end{aligned}
$$

that is,

$$
\begin{aligned}
& \frac{1}{l_{1}} \sum_{\left[\gamma_{j}^{L}, \gamma_{j}^{U}\right] \in \tilde{h}_{j}}\left(\frac{\prod_{j=1}^{n}\left(1+\gamma_{j}^{L}\right)^{\omega_{j}}-\prod_{j=1}^{n}\left(1-\gamma_{j}^{L}\right)^{\omega_{j}}}{\prod_{j=1}^{n}\left(1+\gamma_{j}^{L}\right)^{\omega_{j}}+\prod_{j=1}^{n}\left(1-\gamma_{j}^{L}\right)^{\omega_{j}}}\right. \\
& \left.+\frac{\prod_{j=1}^{n}\left(1+\gamma_{j}^{U}\right)^{\omega_{j}}-\prod_{j=1}^{n}\left(1-\gamma_{j}^{U}\right)^{\omega_{j}}}{\prod_{j=1}^{n}\left(1+\gamma_{j}^{U}\right)^{\omega_{j}}+\prod_{j=1}^{n}\left(1-\gamma_{j}^{U}\right)^{\omega_{j}}}\right) \\
& -\frac{1}{l_{2}} \sum_{\left[\eta_{j}^{L}, \eta_{j}^{U}\right] \in \widetilde{g}_{j}}\left(\frac{2 \prod_{j=1}^{n}\left(\eta_{j}^{L}\right)^{\omega_{j}}}{\prod_{j=1}^{n}\left(2-\eta_{j}^{L}\right)^{\omega_{j}}+\prod_{j=1}^{n}\left(\eta_{j}^{L}\right)^{\omega_{j}}}\right. \\
& \left.+\frac{2 \prod_{j=1}^{n}\left(\eta_{j}^{U}\right)^{\omega_{j}}}{\prod_{j=1}^{n}\left(2-\eta_{j}^{U}\right)^{\omega_{j}}+\prod_{j=1}^{n}\left(\eta_{j}^{U}\right)^{\omega_{j}}}\right) \\
& \leq \frac{1}{l_{3}} \sum_{\left[\gamma_{j}^{L}, \gamma_{j}^{U}\right] \in \widetilde{h}_{j}}\left(1-\prod_{j=1}^{n}\left(1-\gamma_{j}^{L}\right)^{w_{j}}+1-\prod_{j=1}^{n}\left(1-\gamma_{j}^{U}\right)^{w_{j}}\right) \\
& -\frac{1}{l_{4}} \sum_{\left[\eta_{j}^{L}, \eta_{j}^{U}\right] \in \widetilde{g}_{j}}\left(\prod_{j=1}^{n}\left(\eta_{j}^{L}\right)^{\omega_{j}}+\prod_{j=1}^{n}\left(\eta_{j}^{U}\right)^{\omega_{j}}\right),
\end{aligned}
$$

where $l_{1}$ and $l_{2}$ are the numbers of interval values in the membership degrees and nonmembership degrees of $\operatorname{IVDHFEWA}\left(\widetilde{e}_{1}, \widetilde{e}_{2}, \ldots, \widetilde{e}_{n}\right)$, respectively; $l_{3}$ and $l_{4}$ are the numbers of interval values in the membership degrees and nonmembership degrees of $\operatorname{IVDHFWA}\left(\widetilde{e}_{1}, \widetilde{e}_{2}, \ldots, \widetilde{e}_{n}\right)$, respectively. Therefore, according to Theorem 8 , we obtain

$$
\operatorname{IVDHFEWA}\left(\widetilde{e}_{1}, \widetilde{e}_{2}, \ldots, \widetilde{e}_{n}\right) \leq \operatorname{IVDHFWA}\left(\widetilde{e}_{1}, \widetilde{e}_{2}, \ldots, \widetilde{e}_{n}\right) \text {. }
$$

The proof is completed.

Definition 19. Let $\tilde{e}_{j}(j=1,2, \ldots, n)$ be a collection of IVDHFEs; an interval-valued dual hesitant fuzzy Einstein weighted geometric (IVDHFEWG) operator is a mapping IVDHFEWG: $\widetilde{E}^{n} \rightarrow \widetilde{E}$, such that

$\operatorname{IVDHFEWG}\left(\widetilde{e}_{1}, \widetilde{e}_{2}, \ldots, \widetilde{e}_{n}\right)$

$$
\begin{aligned}
= & \bigotimes_{j=1}^{n} \widetilde{e}_{j}^{\omega_{j}} \\
= & \left\{\left\{\left[\frac{2 \prod_{j=1}^{n}\left(\gamma_{j}^{L}\right)^{\omega_{j}}}{\prod_{j=1}^{n}\left(2-\gamma_{j}^{L}\right)^{\omega_{j}}+\prod_{j=1}^{n}\left(\gamma_{j}^{L}\right)^{\omega_{j}}}, \frac{2 \prod_{j=1}^{n}\left(\gamma_{j}^{U}\right)^{\omega_{j}}}{\left.\prod_{j=1}^{n}\left(2-\tilde{h}_{j}, \eta_{j}^{L}, \eta_{j}^{U}\right] \in \tilde{g}_{j}\right)^{\omega_{j}}+\prod_{j=1}^{n}\left(\gamma_{j}^{U}\right)^{\omega_{j}}}\right]\right\},\right. \\
& \left.\left\{\left[\frac{\prod_{j=1}^{n}\left(1+\eta_{j}^{L}\right)^{\omega_{j}}-\prod_{j=1}^{n}\left(1-\eta_{j}^{L}\right)^{\omega_{j}}}{\prod_{j=1}^{n}\left(1+\eta_{j}^{L}\right)^{\omega_{j}}+\prod_{j=1}^{n}\left(1-\eta_{j}^{L}\right)^{\omega_{j}}}, \frac{\prod_{j=1}^{n}\left(1+\eta_{j}^{U}\right)^{\omega_{j}}-\prod_{j=1}^{n}\left(1-\eta_{j}^{U}\right)^{\omega_{j}}+\prod_{j=1}^{n}\left(1-\eta_{j}^{U}\right)^{\omega_{j}}}{\prod_{j=1}^{\omega_{j}}}\right]\right\}\right\},
\end{aligned}
$$

where $\omega=\left(\omega_{1}, \omega_{2}, \ldots, \omega_{n}\right)^{T}$ is the weight vector of $\left(\widetilde{e}_{1}, \widetilde{e}_{2}\right.$, $\left.\ldots, \widetilde{e}_{n}\right)$ with $\omega_{j} \in[0,1]$ and $\sum_{j=1}^{n} \omega_{j}=1$. 
Especially, if $\omega=(1 / n, 1 / n, \ldots, 1 / n)^{T}$, then the IVDHFEWG operator is reduced to an interval-valued dual hesitant fuzzy Einstein geometric (IVDHFEG) operator. Consider

$$
\begin{aligned}
& \operatorname{IVDHFEG}\left(\widetilde{e}_{1}, \widetilde{e}_{2}, \ldots, \widetilde{e}_{n}\right) \\
& =\bigotimes_{j=1}^{n} \widetilde{e}_{j}^{1 / n} \\
& =\bigcup_{\left.\left[\gamma_{j}^{L}, \gamma_{j}^{U}\right] \in \breve{h}_{j}, \eta_{j}^{L}, \eta_{j}^{U}\right] \in \widetilde{g}_{j}}\left\{\left\{\left[\frac{2 \prod_{j=1}^{n}\left(\gamma_{j}^{L}\right)^{1 / n}}{\left.\left.\prod_{j=1}^{n}\left(2-\gamma_{j}^{L}\right)^{1 / n}+\prod_{j=1}^{n}\left(\gamma_{j}^{L}\right)^{1 / n}, \frac{2 \prod_{j=1}^{n}\left(\gamma_{j}^{U}\right)^{1 / n}}{\prod_{j=1}^{n}\left(2-\gamma_{j}^{U}\right)^{1 / n}+\prod_{j=1}^{n}\left(\gamma_{j}^{U}\right)^{1 / n}}\right]\right\},}\right.\right.\right. \\
& \left.\left\{\left[\frac{\prod_{j=1}^{n}\left(1+\eta_{j}^{L}\right)^{1 / n}-\prod_{j=1}^{n}\left(1-\eta_{j}^{L}\right)^{1 / n}}{\prod_{j=1}^{n}\left(1+\eta_{j}^{L}\right)^{1 / n}+\prod_{j=1}^{n}\left(1-\eta_{j}^{L}\right)^{1 / n}}, \frac{\prod_{j=1}^{n}\left(1+\eta_{j}^{U}\right)^{1 / n}-\prod_{j=1}^{n}\left(1-\eta_{j}^{U}\right)^{1 / n}}{\prod_{j=1}^{n}\left(1+\eta_{j}^{U}\right)^{1 / n}+\prod_{j=1}^{n}\left(1-\eta_{j}^{U}\right)^{1 / n}}\right]\right\}\right\} .
\end{aligned}
$$

Similar to the IVDHFEWA operator, the IVDHFEWG operator also has the properties of idempotency and boundedness.

Theorem 20. Let $\tilde{e}_{j}(j=1,2, \ldots, n)$ be a collection of IVDHFEs and let $\omega=\left(\omega_{1}, \omega_{2}, \ldots, \omega_{n}\right)^{T}$ be the weight vector of $\left(\widetilde{e}_{1}, \widetilde{e}_{2}, \ldots, \widetilde{e}_{n}\right)$ with $\omega_{j} \in[0,1]$ and $\sum_{j=1}^{n} \omega_{j}=1$; then

$$
\operatorname{IVDHFWG}\left(\widetilde{e}_{1}, \widetilde{e}_{2}, \ldots, \widetilde{e}_{n}\right) \leq \operatorname{IVDHFEWG}\left(\widetilde{e}_{1}, \widetilde{e}_{2}, \ldots, \widetilde{e}_{n}\right) .
$$

This theorem can be proved similar to Theorem 18 .
3.3. Interval-Valued Dual Hesitant Fuzzy Einstein Ordered Weighted Aggregation Operators. Motivated by the idea of the ordered weighted averaging (OWA) [43] and the ordered weighted geometric [44] operators, we develop some interval-valued dual hesitant fuzzy Einstein ordered weighted aggregation operators.

Definition 21. Let $\widetilde{e}_{j}(j=1,2, \ldots, n)$ be a collection of IVDHFEs, let $\widetilde{e}_{\sigma(j)}$ be the jth largest of them, and let $w=$ $\left(w_{1}, w_{2}, \ldots, w_{n}\right)^{T}$ be the aggregation-associated weight vector, such that $w_{j} \in[0,1]$ and $\sum_{j=1}^{n} w_{j}=1$; then an intervalvalued dual hesitant fuzzy Einstein ordered weighted averaging (IVDHFEOWA) operator is a mapping IVDHFEOWA: $\widetilde{E}^{n} \rightarrow \widetilde{E}$, where

$$
\begin{aligned}
& \operatorname{IVDHFEOWA}\left(\widetilde{e}_{1}, \widetilde{e}_{2}, \ldots, \widetilde{e}_{n}\right) \\
& =\bigoplus_{j=1}^{n}\left(w_{j} \widetilde{e}_{\sigma(j)}\right) \\
& =\bigcup_{\left[\gamma_{\sigma(j)}^{L}, \gamma_{\sigma(j)}^{U}\right] \in \tilde{h}_{\sigma(j)},\left[\eta_{\sigma(j)}^{L}, \eta_{\sigma(j)}^{U}\right] \in \tilde{g}_{\sigma(j)}}\left\{\left\{\left[\frac{\prod_{j=1}^{n}\left(1+\gamma_{\sigma(j)}^{L}\right)^{w_{j}}-\prod_{j=1}^{n}\left(1-\gamma_{\sigma(j)}^{L}\right)^{w_{j}}}{\prod_{j=1}^{n}\left(1+\gamma_{\sigma(j)}^{L}\right)^{w_{j}}+\prod_{j=1}^{n}\left(1-\gamma_{\sigma(j)}^{L}\right)^{w_{j}}}, \frac{\prod_{j=1}^{n}\left(1+\gamma_{\sigma(j)}^{U}\right)^{w_{j}}-\prod_{j=1}^{n}\left(1-\gamma_{\sigma(j)}^{U}\right)^{w_{j}}}{\prod_{j=1}^{n}\left(1+\gamma_{\sigma(j)}^{U}\right)^{w_{j}}+\prod_{j=1}^{n}\left(1-\gamma_{\sigma(j)}^{U}\right)^{w_{j}}}\right]\right\},\right. \\
& \left\{\left[\frac{2 \prod_{j=1}^{n}\left(\eta_{\sigma(j)}^{L}\right)^{w_{j}}}{\prod_{j=1}^{n}\left(2-\eta_{\sigma(j)}^{L}\right)^{w_{j}}+\prod_{j=1}^{n}\left(\eta_{\sigma(j)}^{L}\right)^{w_{j}}}, \frac{2 \prod_{j=1}^{n}\left(\eta_{\sigma(j)}^{U}\right)^{w_{j}}}{\prod_{j=1}^{n}\left(2-\eta_{\sigma(j)}^{U}\right)^{w_{j}}+\prod_{j=1}^{n}\left(\eta_{\sigma(j)}^{U}\right)^{w_{j}}}\right]\right\},
\end{aligned}
$$

where $(\sigma(1), \sigma(2), \ldots, \sigma(n))$ is a permutation of $(1,2, \ldots, n)$, such that $\widetilde{e}_{\sigma(j-1)} \geq \widetilde{e}_{\sigma(j)}$ for all $j=2,3, \ldots, n$.
Especially, if $w=(1 / n, 1 / n, \ldots, 1 / n)^{T}$, then the IVDHFEOWA operator reduces to the IVDHFEA operator in (23). 
Similar to IVDHFEWA and IVDHFEWG operators, the IVDHFEOWA operator also has the properties of idempotency and boundedness. In addition, it has the property of commutativity shown as follows.

Theorem 22 (commutativity). Let $\left(\widetilde{e}_{1}, \widetilde{e}_{2}, \ldots, \widetilde{e}_{n}\right)$ be a collection of IVDHFEs and let $\left(\vec{e}_{1}^{-}, \vec{e}_{2}, \ldots, \vec{e}_{n}^{-}\right)$be any permutation of $\left(\widetilde{e}_{1}, \widetilde{e}_{2}, \ldots, \widetilde{e}_{n}\right)$; then

$$
\begin{aligned}
& \operatorname{IVDHFEOWA}\left(\tilde{e}_{1}, \tilde{e}_{2}, \ldots, \tilde{e}_{n}\right) \\
& \quad=\operatorname{IVDHFEOWA}\left(\vec{e}_{1}, \vec{e}_{2}, \ldots, \widetilde{e}_{n}^{\prime}\right) .
\end{aligned}
$$

Proof. Since $\left(\widetilde{e}_{1}^{\prime}, \widetilde{e}_{2}, \ldots, \widetilde{e}_{n}^{\prime}\right)$ is a permutation of $\left(\widetilde{e}_{1}, \widetilde{e}_{2}, \ldots, \widetilde{e}_{n}\right)$, we have $\widetilde{e}_{\sigma(j)}=\widetilde{e}_{\sigma(j)}$ for all $j=1,2, \ldots, n$. Then, based on Definition 21, we obtain

$$
\begin{aligned}
& \operatorname{IVDHFEOWA}\left(\widetilde{e}_{1}, \widetilde{e}_{2}, \ldots, \widetilde{e}_{n}\right) \\
& \quad=\operatorname{IVDHFEOWA}\left(\widetilde{e}_{1}, \widetilde{e}_{2}, \ldots, \widetilde{e}_{n}^{\prime}\right) .
\end{aligned}
$$

The proof is completed.
Theorem 23. Let $\widetilde{e}_{j}(j=1,2, \ldots, n)$ be a collection of IVDHFEs and let $w=\left(w_{1}, w_{2}, \ldots, w_{n}\right)^{T}$ be the aggregationassociated weight vector, such that $w_{j}>0$ and $\sum_{j=1}^{n} w_{j}=1$; then

$\operatorname{IVDHFEOWA}\left(\widetilde{e}_{1}, \widetilde{e}_{2}, \ldots, \widetilde{e}_{n}\right) \leq \operatorname{IVDHFOWA}\left(\widetilde{e}_{1}, \widetilde{e}_{2}, \ldots, \widetilde{e}_{n}\right)$.

This theorem can be proved similar to Theorem 18 .

Definition 24. Let $\tilde{e}_{j}(j=1,2, \ldots, n)$ be a collection of IVDHFEs, let $\widetilde{e}_{\sigma(j)}$ be the jth largest of them, and let $w=$ $\left(w_{1}, w_{2}, \ldots, w_{n}\right)^{T}$ be the aggregation-associated weight vector, such that $w_{j} \in[0,1]$ and $\sum_{j=1}^{n} w_{j}=1$; then an intervalvalued dual hesitant fuzzy Einstein ordered weighted geometric (IVDHFEOWG) operator is a mapping IVDHFEOWG: $\widetilde{E}^{n} \rightarrow \widetilde{E}$, where

$\operatorname{IVDHFEOWG}\left(\widetilde{e}_{1}, \widetilde{e}_{2}, \ldots, \widetilde{e}_{n}\right)$

$$
\begin{aligned}
& =\bigotimes_{j=1}^{n} \tilde{e}_{\sigma(j)}^{w_{j}} \\
& =\bigcup_{\left[\gamma_{\sigma(j)}^{L}, \gamma_{\sigma(j)}^{U}\right] \in \tilde{h}_{\sigma(j)},\left[\eta_{\sigma(j)}^{L}, \eta_{\sigma(j)}^{U}\right] \in \widetilde{g}_{\sigma(j)}}\left\{\left\{\left[\frac{2 \prod_{j=1}^{n}\left(\gamma_{\sigma(j)}^{L}\right)^{w_{j}}}{\prod_{j=1}^{n}\left(2-\gamma_{\sigma(j)}^{L}\right)^{w_{j}}+\prod_{j=1}^{n}\left(\gamma_{\sigma(j)}^{L}\right)^{w_{j}}}, \frac{2 \prod_{j=1}^{n}\left(\gamma_{\sigma(j)}^{U}\right)^{w_{j}}}{\prod_{j=1}^{n}\left(2-\gamma_{\sigma(j)}^{U}\right)^{w_{j}}+\prod_{j=1}^{n}\left(\gamma_{\sigma(j)}^{U}\right)^{w_{j}}}\right]\right\},\right. \\
& \left.\left\{\left[\frac{\prod_{j=1}^{n}\left(1+\eta_{\sigma(j)}^{L}\right)^{w_{j}}-\prod_{j=1}^{n}\left(1-\eta_{\sigma(j)}^{L}\right)^{w_{j}}}{\prod_{j=1}^{n}\left(1+\eta_{\sigma(j)}^{L}\right)^{w_{j}}+\prod_{j=1}^{n}\left(1-\eta_{\sigma(j)}^{L}\right)^{w_{j}}}, \frac{\prod_{j=1}^{n}\left(1+\eta_{\sigma(j)}^{U}\right)^{w_{j}}-\prod_{j=1}^{n}\left(1-\eta_{\sigma(j)}^{U}\right)^{w_{j}}}{\prod_{j=1}^{n}\left(1+\eta_{\sigma(j)}^{U}\right)^{w_{j}}+\prod_{j=1}^{n}\left(1-\eta_{\sigma(j)}^{U}\right)^{w_{j}}}\right]\right\}\right\}
\end{aligned}
$$

where $(\sigma(1), \sigma(2), \ldots, \sigma(n))$ is a permutation of $(1,2, \ldots, n)$, such that $\widetilde{e}_{\sigma(j-1)} \geq \widetilde{e}_{\sigma(j)}$ for all $j=2,3, \ldots, n$.

Especially, if $w=(1 / n, 1 / n, \ldots, 1 / n)^{T}$, then the IVDHFEOWG operator reduces to the IVDHFEG operator in (49).

Theorem 25. Let $\tilde{e}_{j}(j=1,2, \ldots, n)$ be a collection of IVDHFEs and let $w=\left(w_{1}, w_{2}, \ldots, w_{n}\right)^{T}$ be the aggregationassociated weight vector, such that $w_{j} \in[0,1]$ and $\sum_{j=1}^{n} w_{j}=1$; then

$$
\begin{aligned}
& \operatorname{IVDHFOWG}\left(\widetilde{e}_{1}, \widetilde{e}_{2}, \ldots, \widetilde{e}_{n}\right) \\
& \quad \leq \operatorname{IVDHFEOWG}\left(\widetilde{e}_{1}, \widetilde{e}_{2}, \ldots, \widetilde{e}_{n}\right) .
\end{aligned}
$$

This theorem can be proved similar to Theorem 18 .
3.4. Interval-Valued Dual Hesitant Fuzzy Hybrid Aggregation Operators Based on Einstein Operations. From Definitions 14-24, we can see that the IVDHFEWA and IVDHFEWG operators only weight the importance of interval-valued dual hesitant fuzzy argument itself, while the IVDHFEOWA and IVDHFEOWG operators only weight the importance of ordered position of each argument. Therefore, weights represent different aspects in both weighted aggregation (IVDHFEWA and IVDHFEWG) operators and ordered weighted aggregation (IVDHFEOWA and IVDHFEOWG) operators. To solve this drawback, in what follows, we will propose some interval-valued dual hesitant fuzzy Einstein hybrid aggregation operators, which weight both the given interval-valued dual hesitant fuzzy arguments and their ordered positions. Motivated by the hybrid aggregation operators [45], which consider both the given arguments and their ordered positions, in what follows, we will propose some interval-valued dual hesitant fuzzy Einstein hybrid aggregation operators. 
Definition 26. Let $\tilde{e}_{j}(j=1,2, \ldots, n)$ be a collection of IVDHFEs, let $\omega=\left(\omega_{1}, \omega_{2}, \ldots, \omega_{n}\right)^{T}$ be the weight vector of $\widetilde{e}_{j}(j=1,2, \ldots, n)$ with $\omega_{j} \in[0,1], \sum_{j=1}^{n} \omega_{j}=1$, and let $n$ be the balancing coefficient which plays a role of balance; then an interval-valued dual hesitant fuzzy Einstein hybrid averaging (IVDHFEHA) operator is a mapping $\widetilde{E}^{n} \rightarrow \widetilde{E}$ with the aggregation-associated weight vector $w=\left(w_{1}, w_{2}, \ldots, w_{n}\right)^{T}$, such that $w_{j} \in[0,1]$ and $\sum_{j=1}^{n} w_{j}=1$ :

$$
\begin{aligned}
& \operatorname{IVDHFEHA}\left(\widetilde{e}_{1}, \widetilde{e}_{2}, \ldots, \widetilde{e}_{n}\right) \\
& =\bigoplus_{j=1}^{n}\left(w_{j} \dot{e}_{\sigma(j)}\right) \\
& =\bigcup_{\left[\dot{\gamma}_{\sigma(j)}^{L}, \dot{\gamma}_{\sigma(j)}^{U}\right] \in \dot{h}_{\sigma(j)},\left[\dot{\eta}_{\sigma(j)}^{L}, \dot{\eta}_{\sigma(j)}^{U}\right] \in \dot{g}_{\sigma(j)}}\left\{\left\{\left[\frac{\prod_{j=1}^{n}\left(1+\dot{\gamma}_{\sigma(j)}^{L}\right)^{w_{j}}-\prod_{j=1}^{n}\left(1-\dot{\gamma}_{\sigma(j)}^{L}\right)^{w_{j}}}{\prod_{j=1}^{n}\left(1+\dot{\gamma}_{\sigma(j)}^{L}\right)^{w_{j}}+\prod_{j=1}^{n}\left(1-\dot{\gamma}_{\sigma(j)}^{L}\right)^{w_{j}}}, \frac{\prod_{j=1}^{n}\left(1+\dot{\gamma}_{\sigma(j)}^{U}\right)^{w_{j}}-\prod_{j=1}^{n}\left(1-\dot{\gamma}_{\sigma(j)}^{U}\right)^{w_{j}}}{\prod_{j=1}^{n}\left(1+\dot{\gamma}_{\sigma(j)}^{U}\right)^{w_{j}}+\prod_{j=1}^{n}\left(1-\dot{\gamma}_{\sigma(j)}^{U}\right)^{w_{j}}}\right]\right\},\right. \\
& \left.\left\{\left[\frac{2 \prod_{j=1}^{n}\left(\dot{\eta}_{\sigma(j)}^{L}\right)^{w_{j}}}{\prod_{j=1}^{n}\left(2-\dot{\eta}_{\sigma(j)}^{L}\right)^{w_{j}}+\prod_{j=1}^{n}\left(\dot{\eta}_{\sigma(j)}^{L}\right)^{w_{j}}}, \frac{2 \prod_{j=1}^{n}\left(\dot{\eta}_{\sigma(j)}^{U}\right)^{w_{j}}}{\prod_{j=1}^{n}\left(2-\dot{\eta}_{\sigma(j)}^{U}\right)^{w_{j}}+\prod_{j=1}^{n}\left(\dot{\eta}_{\sigma(j)}^{U}\right)^{w_{j}}}\right]\right\}\right\},
\end{aligned}
$$

where $\dot{e}_{\sigma(j)}$ is the $j$ th largest of interval-valued dual hesitant fuzzy weighted arguments $\dot{e}_{i}\left(\dot{e}_{i}=n \omega_{i} \widetilde{e}_{i}\right),(i=1,2, \ldots, n)$. $\omega=\left(\omega_{1}, \omega_{2}, \ldots, \omega_{n}\right)^{T}$ is the weight vector of $\widetilde{e}_{j}$ and $w=$ $\left(w_{1}, w_{2}, \ldots, w_{n}\right)^{T}$ is the aggregation-associated weight vector, such that $w_{j} \in[0,1]$ and $\sum_{j=1}^{n} w_{j}=1$.

Especially, if $w=(1 / n, 1 / n, \ldots, 1 / n)^{T}$, then the IVDHFEHA operator reduces to the IVDHFEWA operator in (16). If $\omega=(1 / n, 1 / n, \ldots, 1 / n)^{T}$, then the IVDHFEHA operator reduces to the IVDHFEOWA operator in (51).

Theorem 27. Let $\tilde{e}_{j}(j=1,2, \ldots, n)$ be a collection of IVDHFEs, let $\omega=\left(\omega_{1}, \omega_{2}, \ldots, \omega_{n}\right)^{T}$ be the weight vector of $\tilde{e}_{j}$ $(j=1,2, \ldots, n)$, with $\omega_{j} \in[0,1], \sum_{j=1}^{n} \omega_{j}=1$, and let $w=\left(w_{1}, w_{2}, \ldots, w_{n}\right)^{T}$ be the aggregation-associated weight vector, such that $w_{j} \in[0,1]$ and $\sum_{j=1}^{n} w_{j}=1$; then

$$
\operatorname{IVDHFEHA}\left(\widetilde{e}_{1}, \widetilde{e}_{2}, \ldots, \widetilde{e}_{n}\right) \leq \operatorname{IVDHFHA}\left(\widetilde{e}_{1}, \tilde{e}_{2}, \ldots, \widetilde{e}_{n}\right) \text {. }
$$

This theorem can be proved similar to Theorem 18.

Definition 28. Let $\tilde{e}_{j}(j=1,2, \ldots, n)$ be a collection of IVDHFEs, let $\omega=\left(\omega_{1}, \omega_{2}, \ldots, \omega_{n}\right)^{T}$ be the weight vector of $\tilde{e}_{j}$ $(j=1,2, \ldots, n)$, with $\omega_{j} \in[0,1], \sum_{j=1}^{n} \omega_{j}=1$, and let $n$ be the balancing coefficient which plays a role of balance; then an interval-valued dual hesitant fuzzy Einstein hybrid geometric (IVDHFEHG) operator is a mapping $\widetilde{E}^{n} \rightarrow \widetilde{E}$ with the aggregation-associated weight vector $w=\left(w_{1}, w_{2}, \ldots, w_{n}\right)^{T}$, such that $w_{j} \in[0,1]$ and $\sum_{j=1}^{n} w_{j}=1$ :

\section{$\operatorname{IVDHFEHG}\left(\widetilde{e}_{1}, \widetilde{e}_{2}, \ldots, \widetilde{e}_{n}\right)$}

$$
\begin{aligned}
& =\bigotimes_{j=1}^{n} \ddot{e}_{\sigma(j)}^{w_{j}} \\
& =\bigcup_{\left[\ddot{\gamma}_{\sigma(j)}^{L}, \ddot{\gamma}_{\sigma(j)}^{U}\right] \in \ddot{h}_{j},\left[\ddot{\eta}_{\sigma(j)}^{L}, \ddot{\eta}_{\sigma(j)}^{U}\right] \in \ddot{g}_{j}}\left\{\left\{\left[\frac{2 \prod_{j=1}^{n}\left(\ddot{\gamma}_{\sigma(j)}^{L}\right)^{w_{j}}}{\prod_{j=1}^{n}\left(2-\ddot{\gamma}_{\sigma(j)}^{L}\right)^{w_{j}}+\prod_{j=1}^{n}\left(\ddot{\gamma}_{\sigma(j)}^{L}\right)^{w_{j}}}, \frac{2 \prod_{j=1}^{n}\left(\ddot{\gamma}_{\sigma(j)}^{U}\right)^{w_{j}}}{\prod_{j=1}^{n}\left(2-\ddot{\gamma}_{\sigma(j)}^{U}\right)^{w_{j}}+\prod_{j=1}^{n}\left(\ddot{\gamma}_{\sigma(j)}^{U}\right)^{w_{j}}}\right]\right\},\right. \\
& \left\{\left[\frac{\prod_{j=1}^{n}\left(1+\ddot{\eta}_{\sigma(j)}^{L}\right)^{w_{j}}-\prod_{j=1}^{n}\left(1-\ddot{\eta}_{\sigma(j)}^{L}\right)^{w_{j}}}{\prod_{j=1}^{n}\left(1+\ddot{\eta}_{\sigma(j)}^{L}\right)^{w_{j}}+\prod_{j=1}^{n}\left(1-\ddot{\eta}_{\sigma(j)}^{L}\right)^{w_{j}}}, \frac{\prod_{j=1}^{n}\left(1+\ddot{\eta}_{\sigma(j)}^{U}\right)^{w_{j}}-\prod_{j=1}^{n}\left(1-\ddot{\eta}_{\sigma(j)}^{U}\right)^{w_{j}}}{\prod_{j=1}^{n}\left(1+\ddot{\eta}_{\sigma(j)}^{U}\right)^{w_{j}}+\prod_{j=1}^{n}\left(1-\ddot{\eta}_{\sigma(j)}^{U}\right)^{w_{j}}}\right]\right\},
\end{aligned}
$$


where $\ddot{e}_{\sigma(j)}$ is the $j$ th largest of interval-valued dual hesitant fuzzy weighted arguments $\ddot{e}_{i}\left(\ddot{e}_{i}=\widetilde{e}_{i}^{n \omega_{i}}\right),(i=1,2, \ldots, n)$. $\omega=\left(\omega_{1}, \omega_{2}, \ldots, \omega_{n}\right)^{T}$ is the weight vector of $\tilde{e}_{j}$ and $w=$ $\left(w_{1}, w_{2}, \ldots, w_{n}\right)^{T}$ is the aggregation-associated weight vector, such that $w_{j} \in[0,1]$ and $\sum_{j=1}^{n} w_{j}=1$.

Especially, if $w=(1 / n, 1 / n, \ldots, 1 / n)^{T}$, then the IVDHFEHG operator reduces to the IVDHFEWG operator in (49). If $\omega=(1 / n, 1 / n, \ldots, 1 / n)^{T}$, then the IVDHFEHG operator reduces to the IVDHFEOWG operator in (55).

Theorem 29. Let $\widetilde{e}_{j}(j=1,2, \ldots, n)$ be a collection of IVDHFEs, let $\omega=\left(\omega_{1}, \omega_{2}, \ldots, \omega_{n}\right)^{T}$ be the weight vector of $\widetilde{e}_{j}(j=$ $1,2, \ldots, n)$, with $\omega_{j} \in[0,1], \sum_{j=1}^{n} \omega_{j}=1$, and let $w=\left(w_{1}, w_{2}\right.$, $\left.\ldots, w_{n}\right)^{T}$ be the aggregation-associated weight vector, such that $w_{j} \in[0,1]$ and $\sum_{j=1}^{n} w_{j}=1 ;$ then

$$
\operatorname{IVDHFHG}\left(\widetilde{e}_{1}, \widetilde{e}_{2}, \ldots, \widetilde{e}_{n}\right) \leq \operatorname{IVDHFEHG}\left(\widetilde{e}_{1}, \tilde{e}_{2}, \ldots, \widetilde{e}_{n}\right) \text {. }
$$

This theorem can be proved similar to Theorem 18 .

\section{An Approach to MADM with Interval-Valued Dual Hesitant Fuzzy Information}

In this section, we apply the aggregation operators proposed above to multiattribute decision making with interval-valued dual hesitant fuzzy information. Let $A=\left\{A_{1}, A_{2}, \ldots, A_{m}\right\}$ be a finite set of $m$ alternatives, let $C=\left\{C_{1}, C_{2}, \ldots, C_{n}\right\}$ be the set of $n$ attributes, and let $\omega=\left(\omega_{1}, \omega_{2}, \ldots, \omega_{n}\right)^{T}$ be the weight vector of attributes $C_{j}(j=1,2, \ldots, n)$ with $\omega_{j} \in[0,1]$ and $\sum_{j=1}^{n} \omega_{j}=1$. Suppose that $\widetilde{E}=\left(\widetilde{e}_{i j}\right)_{m \times n}$ is an intervalvalued dual hesitant fuzzy matrix, where $\widetilde{e}_{i j}=\left\{\widetilde{h}_{i j}, \widetilde{g}_{i j}\right\}$ is in the form of IVDHFE given for alternative $A_{i}(i=1,2, \ldots, m)$ with respect to attribute $C_{j}(j=1,2, \ldots, n)$, with $\widetilde{h}_{i j}=$ $\bigcup_{\left[\gamma_{i j}^{L}, \gamma_{i j}^{U}\right] \in \widetilde{h}_{i j}}\left\{\left[\gamma_{i j}^{L}, \gamma_{i j}^{U}\right]\right\}$ and $\widetilde{g}_{i j}=\bigcup_{\left[\eta_{i j}^{L}, \eta_{i j}^{U}\right] \in \widetilde{g}_{i j}}\left\{\left[\eta_{i j}^{L}, \eta_{i j}^{U}\right]\right\}$. Then, to determine the most desirable alternative(s), the IVDHFEWA operator is utilized to develop a multiattribute decision making method with interval-valued dual hesitant fuzzy information by the following steps.

Step 1. Obtain the interval-valued dual hesitant fuzzy matrix. The decision makers provide their evaluations about alternative $A_{i}$ under attribute $C_{j}$, denoted by the intervalvalued dual hesitant fuzzy elements $\widetilde{e}_{i j}=\left\{\widetilde{h}_{i j}, \widetilde{g}_{i j}\right\}=$ $\left\{\bigcup_{\left[\gamma_{i j}^{L}, \gamma_{i j}^{U}\right] \in \tilde{h}_{i j}}\left\{\left[\gamma_{i j}^{L}, \gamma_{i j}^{U}\right]\right\}, \bigcup_{\left[\eta_{i j}^{L}, \eta_{i j}^{U}\right] \in \tilde{g}_{i j}}\left\{\left[\eta_{i j}^{L}, \eta_{i j}^{U}\right]\right\}\right\},(i=1,2, \ldots, m$; $j=1,2, \ldots, n)$.

Step 2. Compute overall assessments of alternatives. Utilize the IVDHFEWA operator to aggregate all the rating values $\widetilde{e}_{i j}(j=1,2, \ldots, n)$ of the $i$ th line and get the overall rating value $\widetilde{e}_{i}$ corresponding to alternative $A_{i}(i=1,2, \ldots, m)$; that is,

$$
\begin{aligned}
& \tilde{e}_{i}=\operatorname{IVDHFEWA}\left(\tilde{e}_{i 1}, \tilde{e}_{i 2}, \ldots, \tilde{e}_{i n}\right) \\
&=\bigoplus_{j=1}^{n} \omega_{j} \widetilde{e}_{i j} \\
&=\bigcup_{\left[\gamma_{i j}^{L}, \gamma_{i j}^{U}\right] \in \widetilde{h}_{i j},\left[\eta_{i j}^{L}, \eta_{i j}^{U}\right] \in \tilde{g}_{i j}}\left\{\left\{\frac{\prod_{j=1}^{n}\left(1+\gamma_{i j}^{L}\right)^{\omega_{j}}-\prod_{j=1}^{n}\left(1-\gamma_{i j}^{L}\right)^{\omega_{j}}}{\prod_{j=1}^{n}\left(1+\gamma_{i j}^{L}\right)^{\omega_{j}}+\prod_{j=1}^{n}\left(1-\gamma_{i j}^{L}\right)^{\omega_{j}}}, \frac{\prod_{j=1}^{n}\left(1+\gamma_{i j}^{U}\right)^{\omega_{j}}-\prod_{j=1}^{n}\left(1-\gamma_{i j}^{U}\right)^{\omega_{j}}}{\prod_{j=1}^{n}\left(1+\gamma_{i j}^{U}\right)^{\omega_{j}}+\prod_{j=1}^{n}\left(1-\gamma_{i j}^{U}\right)^{\omega_{j}}}\right]\right\}, \\
&\left.\left\{\left[\frac{2 \prod_{j=1}^{n}\left(\eta_{i j}^{L}\right)^{\omega_{j}}}{\prod_{j=1}^{n}\left(2-\eta_{i j}^{L}\right)^{\omega_{j}}+\prod_{j=1}^{n}\left(\eta_{i j}^{L}\right)^{\omega_{j}}, \frac{2}{\prod_{j=1}^{n}\left(\eta_{i j}^{U}\right)^{\omega_{j}}}\left(2-\eta_{i j}^{U}\right)^{\omega_{j}}+\prod_{j=1}^{n}\left(\eta_{i j}^{U}\right)^{\omega_{j}}}\right]\right\}\right\},
\end{aligned}
$$

where $\omega=\left(\omega_{1}, \omega_{2}, \ldots, \omega_{n}\right)^{T}$ is the weight vector of attributes $C_{j}(j=1,2, \ldots, n)$, such that $\omega_{j} \in[0,1]$ and $\sum_{j=1}^{n} \omega_{j}=$ 1. Note that $\tilde{e}_{i}$ is in the forms of IVDHFEs, and it can be denoted by $\widetilde{e}_{i}=\left\{\tilde{h}_{i}, \tilde{g}_{i}\right\}, \tilde{h}_{i}=\bigcup_{\left[\gamma_{i}^{L}, \gamma_{i}^{U}\right] \in \tilde{h}_{i}}\left\{\left[\gamma_{i}^{L}, \gamma_{i}^{U}\right]\right\}$, and $\tilde{g}_{i}=$ $\bigcup_{\left[\eta_{i}^{L}, \eta_{i}^{U}\right] \in \tilde{g}_{i}}\left\{\left[\eta_{i}^{L}, \eta_{i}^{U}\right]\right\}$.

Step 3. Compare the score values $S\left(\widetilde{e}_{i}\right)$ of overall assessments values $\widetilde{e}_{i}(i=1,2, \ldots, m)$ using score function by

$$
S\left(\widetilde{e}_{i}\right)=\frac{1}{2}\left(\frac{1}{l\left(\widetilde{h}_{i}\right)} \sum_{\left[\gamma_{i}^{L}, \gamma_{i}^{U}\right] \in \tilde{h}_{i}}\left(\gamma_{i}^{L}+\gamma_{i}^{U}\right)\right.
$$

$$
\left.-\frac{1}{l\left(\widetilde{g}_{i}\right)} \sum_{\left[\eta_{i}^{L}, \eta_{i}^{U}\right] \in \widetilde{g}_{i}}\left(\eta_{i}^{L}+\eta_{i}^{U}\right)\right),
$$

where $l\left(\widetilde{h}_{i}\right)$ and $l\left(\widetilde{g}_{i}\right)$ are the numbers of interval values in $\widetilde{h}_{i}$ and $\widetilde{g}_{i}$, respectively. If $S\left(\widetilde{e}_{i}\right)=S\left(\widetilde{e}_{t}\right)$, then we need to calculate the accuracy values $P\left(\widetilde{e}_{i}\right)$ and $P\left(\widetilde{e}_{t}\right)$ of alternatives $A_{i}$ and $A_{t}(i, t=1,2, \ldots, m)$ by the following:

$$
\begin{aligned}
P\left(\widetilde{e}_{i}\right)=\frac{1}{2}( & \frac{1}{l\left(h_{i}\right)} \sum_{\left[\gamma_{i}^{L}, \gamma_{i}^{U}\right] \in \tilde{h}_{i}}\left(\gamma_{i}^{L}+\gamma_{i}^{U}\right) \\
& \left.+\frac{1}{l\left(\tilde{g}_{i}\right)} \sum_{\left[\eta_{i}^{L}, \eta_{i}^{U}\right] \in \tilde{g}_{i}}\left(\eta_{i}^{L}+\eta_{i}^{U}\right)\right) .
\end{aligned}
$$


Step 4. Rank all feasible alternatives $A_{i}(i=1,2, \ldots, m)$ according to Theorem 8 and select the most desirable alternative(s).

Step 5. End.

\section{Illustrative Example}

5.1. An Example. In this section, a MADM problem adapted from [34] is used to illustrate the developed procedure. In [34], Wang and Lee considered a software selection problem in which the alternatives are the software packages to be selected and the criteria are the attributes under consideration. The manger of a computer center at a university wishes to select a new information system to improve work productivity. After preliminary screening, four alternatives $A_{i}(i=1,2,3,4)$ remain on the candidate list. And three attributes are under consideration: (1) the cost of the hardware/software investment $\left(C_{1}\right),(2)$ the contribution to the performance of the organization $\left(C_{2}\right)$, and (3) the effort to transfer from the current system $\left(C_{3}\right)$. The weight vector of attributes $C_{j}(j=1,2,3)$ is $\omega=(0.35,0.25,0.4)^{T}$. The experts evaluate the software packages $A_{i}(i=1,2,3,4)$ with respect to attributes $C_{j}(j=1,2,3)$ and the evaluations are expressed in the form of IVDHFE. In what follows, we use the MADM method proposed in Section 4 to select the most desirable software package(s).

Step 1. Determine the interval-valued dual hesitant fuzzy matrix $\widetilde{E}=\left(\widetilde{e}_{i j}\right)_{4 \times 3}$ shown in Table 1 , where $\widetilde{e}_{i j}$ is the evaluation value about the alternative $A_{i}$ with respect to the attribute $C_{j}$ and it is in the form of IVDHFE.

Step 2. Utilize (61) to aggregate all the rating values $\widetilde{e}_{i j}$ of alternative $A_{i}(i=1,2,3,4)$ on all attributes $C_{j}(j=1,2,3)$ into overall assessment values $\widetilde{e}_{i}(i=1,2,3,4)$, which are shown as follows:

$$
\begin{aligned}
& \widetilde{e}_{1}=\{\{[0.3000,0.4663],[0.3410,0.4663], \\
& {[0.3410,0.5101],[0.3257,0.4663] \text {, }} \\
& {[0.3659,0.4663],[0.3659,0.5101]} \\
& {[0.3359,0.5000],[0.3758,0.5000] \text {, }} \\
& {[0.3758,0.5419],[0.3610,0.5000] \text {, }} \\
& {[0.4000,0.5000],[0.4000,0.5419] \text {, }} \\
& {[0.3743,0.5368],[0.4129,0.5368] \text {, }} \\
& {[0.4129,0.5765],[0.3986,0.5368] \text {, }} \\
& [0.4363,0.5368],[0.4363,0.5765]\} \text {, } \\
& \{[0.1523,0.3000],[0.2000,0.3000] \text {, }
\end{aligned}
$$

[0.1694, 0.3229], [0.2218, 0.3229],

$[0.1523,0.3324],[0.2000,0.3324]$,

$[0.1694,0.3573],[0.2218,0.3573]\}\} ;$

$\widetilde{e}_{2}=\{\{[0.2975,0.3986],[0.3758,0.4761]$,

[0.4181, 0.5193], [0.2730, 0.3986],

$[0.3526,0.4761],[0.3958,0.5193]$,

$[0.3370,0.4401],[0.4129,0.5141]$,

$[0.4537,0.5551],[0.3131,0.4401]$,

$[0.3905,0.5141],[0.4323,0.5551]\}$,

$\{[0.2311,0.3729],[0.2717,0.3729]$,

$[0.2759,0.4235],[0.3229,0.4235]\}\} ;$

$\widetilde{e}_{3}=\{\{[0.3171,0.4761],[0.3616,0.5193]$,

$[0.4106,0.5684],[0.3659,0.5000]$,

[0.4086, 0.5419], [0.4554, 0.5892],

$[0.3928,0.5265],[0.4345,0.5668]$,

$[0.4799,0.6122],[0.3526,0.4761]$,

$[0.3958,0.5193],[0.4432,0.5684]$,

$[0.4000,0.5000],[0.4414,0.5419]$,

$[0.4865,0.5892],[0.4261,0.5265]$,

$[0.4663,0.5668],[0.5101,0.6122]\}$,

$\{[0.1280,0.3000],[0.1688,0.3000]$,

[0.1523, 0.3229], [0.2000, 0.3229],

$[0.1488,0.3613],[0.1956,0.3613]$,

$[0.1767,0.3878],[0.2311,0.3878]\}\}$

$\widetilde{e}_{4}=\{\{[0.3659,0.5101],[0.4554,0.5598]$,

$[0.3928,0.5362],[0.4799,0.5840]$,

$[0.4000,0.5765],[0.4865,0.6211]$,

[0.4261, 0.6000], [0.5101, 0.6427], 
TABLE 1: Interval-valued dual hesitant fuzzy decision matrix.

\begin{tabular}{cccr}
\hline & $C_{1}$ & $C_{2}$ & $C_{3}$ \\
\hline \multirow{2}{*}{$A_{1}$} & $\{\{[0.3,0.4],[0.4,0.5],[0.5,0.6]\}$, & $\{\{[0.3,0.5],[0.4,0.5]\}$, & $\{\{[0.3,0.5],[0.4,0.5],[0.4,0.6]\}$, \\
& $\{[0.2,0.3],[0.2,0.4]\}\}$ & $\{[0.2,0.3],[0.3,0.4]\}\}$ & $\{[0.1,0.3],[0.2,0.3]\}\}$ \\
\hline \multirow{2}{*}{$A_{2}$} & $\{\{[0.4,0.5],[0.5,0.6]\},\{[0.3,0.4]\}\}$ & $\{\{0.3,0.4],[0.2,0.4]\}$, & $\{\{0.2,0.3],[0.4,0.5],[0.5,0.6]\}$, \\
& $\{\{[0.3,0.5],[0.4,0.5]\}$, & $\{[0.2,0.3],[0.4,0.5]\}\}$ & $\{[0.2,0.4],[0.3,0.4]\}\}$ \\
\multirow{2}{*}{$A_{3}$} & $\{[0.2,0.3],[0.3,0.5]\}\}$ & $\{\{[0.2,0.4],[0.4,0.5],[0.5,0.6]\}$, & $\{\{[0.4,0.5],[0.5,0.6],[0.6,0.7]\}$, \\
& $\{\{[0.3,0.4],[0.4,0.6]$, & $\{[0.1,0.3],[0.2,0.4]\}\}$ & $\{[0.1,0.3],[0.2,0.3]\}\}$ \\
\hline \multirow{2}{*}{$A_{4}$} & $[0.5,0.8]\},\{[0.1,0.2]\}\}$ & $\{\{[0.4,0.5],[0.5,0.6]\}$, & $\{\{[0.4,0.6],[0.6,0.7]\}$, \\
& & $\{[0.2,0.3],[0.2,0.4]\}\}$ & $\{[0.1,0.2],[0.1,0.3]\}\}$ \\
\hline
\end{tabular}

$[0.4363,0.6635],[0.5193,0.7007]$

$[0.4614,0.6832],[0.5419,0.7186]\}$,

$\{[0.1193,0.2218],[0.1193,0.2610]$,

$[0.1193,0.2396],[0.1193,0.2814]\}\}$.

Step 3. Compare the magnitude of the different overall assessments values $\tilde{e}_{i}(i=1,2,3,4)$ using score function according to (62):

$$
\begin{array}{ll}
S\left(\widetilde{e}_{1}\right)=0.1882 ; & S\left(\widetilde{e}_{2}\right)=0.0907 ; \\
S\left(\widetilde{e}_{3}\right)=0.2229 ; & S\left(\widetilde{e}_{4}\right)=0.3512 .
\end{array}
$$

Step 4. Rank all the alternatives $A_{i}(i=1,2,3,4)$ according to Theorem 8 . Since $S\left(\widetilde{e}_{4}\right)>S\left(\widetilde{e}_{3}\right)>S\left(\widetilde{e}_{1}\right)>S\left(\widetilde{e}_{2}\right)$, then the ranking of the alternatives is shown as follows: $A_{4}>A_{3}>$ $A_{1}>A_{2}$. Therefore, the most desirable alternative is $A_{4}$.

5.2. Comparison with Other Methods. To test the validity of the proposed method, the evaluation results in Section 5.1 are compared with that from the other method proposed by Ju et al. [29]. The main difference between the two methods is due to the aggregation process. Specifically, in the process of aggregating the attributes' values, the method proposed in this paper uses the IVDHFEWA operator that is based on the Einstein $t$-norms and $t$-conorms, while the method presented in [29] uses the IVDHFWA operator that is based on the algebraic $t$-norms and $t$-conorms.

Therefore, if we use the IVDHFWA operator instead of the IVDHFEWA operator in Step 2 in Section 5.1, the aggregated results $\widetilde{e}_{i}(i=1,2,3,4)$ with respect to the rating values $\widetilde{e}_{i j}(i=1,2,3,4, j=1,2,3)$ are shown as follows:

$$
\begin{array}{r}
\widetilde{e}_{1}=\{\{[0.3000,0.4671],[0.3419,0.4671], \\
{[0.3419,0.5126],[0.3265,0.4671],} \\
{[0.3667,0.4671],[0.3667,0.5126],}
\end{array}
$$

$[0.3368,0.5000],[0.3764,0.5000]$

$[0.3764,0.5427],[0.3618,0.5000]$

$[0.4000,0.5000],[0.4000,0.5427]$

$[0.3778,0.5376],[0.4150,0.5376]$

$[0.4150,0.5771],[0.4013,0.5736]$

$[0.4371,0.5376],[0.4371,0.5771]\}$,

$\{[0.1516,0.3000],[0.2000,0.3000]$,

$[0.1677,0.3224],[0.2213,0.3224]$,

$[0.1516,0.3318],[0.2000,0.3318]$,

$[0.1677,0.3565],[0.2213,0.3565]\}\} ;$

$\widetilde{e}_{2}=\{\{[0.3004,0.4013],[0.3764,0.4767]$,

$[0.4203,0.5214],[0.2766,0.4013]$,

$[0.3553,0.4767],[0.4006,0.5214]$,

$[0.3436,0.4463],[0.4150,0.5160]$,

$[0.4561,0.5573],[0.3213,0.4463]$,

$[0.3951,0.5160],[0.4377,0.5573]\}$,

$\{[0.2305,0.3722],[0.2711,0.3722]$,

$[0.2741,0.4229],[0.3224,0.4229]\}\}$

$\widetilde{e}_{3}=\{\{[0.3195,0.4767],[0.3674,0.5214]$,

$[0.4214,0.5734],[0.3667,0.5000]$,

$[0.4113,0.5427],[0.4615,0.5924]$,

$[0.3950,0.5271],[0.4375,0.5675]$, 


$$
\begin{gathered}
{[0.4855,0.6145],[0.3553,0.4767],} \\
{[0.4006,0.5214],[0.4518,0.5734],} \\
{[0.4000,0.5000],[0.4422,0.5427],} \\
{[0.4898,0.5924],[0.4267,0.5271],} \\
[0.4671,0.5675],[0.5126,0.6145]\}, \\
\{[0.1275,0.3000],[0.1682,0.3000], \\
\quad[0.1516,0.3224],[0.2000,0.3224], \\
\quad[0.1469,0.3587],[0.1938,0.3587], \\
\quad[0.1747,0.3855],[0.2305,0.3855]\}\} ; \\
{[\{[0.3667,0.5216],[0.4615,0.5655],} \\
{[0.3950,0.5390],[0.4855,0.5891],} \\
{[0.4000,0.5771],[0.4898,0.6230],} \\
{[0.4267,0.6000],[0.5216,0.6435],} \\
{[0.4371,0.6682],[0.5214,0.7042],} \\
[0.4622,0.6862],[0.5427,0.7203]\}, \\
\{[0.1189,0.2213],[0.1189,0.2603], \\
[0.1189,0.2378],[0.1189,0.2797]\}\}
\end{gathered}
$$

Similarly, the score function values of the $\widetilde{e}_{i}(i=1,2,3,4)$ can be calculated according to (62); the results are shown as follows:

$$
\begin{array}{ll}
S\left(\widetilde{e}_{1}\right)=0.1897 ; & S\left(\widetilde{e}_{2}\right)=0.0946 ; \\
S\left(\widetilde{e}_{3}\right)=0.2266 ; & S\left(\widetilde{e}_{4}\right)=0.3544 .
\end{array}
$$

Obviously, the ranking order of the four alternatives is $A_{4}>A_{3}>A_{1}>A_{2}$, which is exactly the same as that obtained in Section 5.1.

It is interesting to point out that the score values obtained by the IVDHFEWA operator are smaller than those obtained by the IVDHFWA operator, which is consistent with Theorem 18.

From the above analysis, we can clearly find that the proposed approach is effective. In addition, when the decision makers show some kind of pessimistic attitude towards the decision making problems, they can choose the IVDHFEWA operator, which has more merits in characterizing the pessimistic attitude than the IVDHFWA operator.

\section{Conclusions}

The traditional dual hesitant fuzzy aggregation operators are generally suitable for aggregating information taking the form of numerical numbers, and yet they will fail in dealing with interval-valued dual hesitant fuzzy information. In this paper, we investigate the MADM problems in which the attribute values take the form of interval-valued dual hesitant fuzzy information. Firstly, we propose some operational laws for IVDHFEs based on Einstein operations. Then, we develop some interval-valued dual hesitant fuzzy Einstein aggregation operators: the IVDHFEWA operator, IVDHFEWG operator, IVDHFEOWA operator, and IVDHFEOWG operator. Some desirable properties of these operators and the relationship between the developed operators and the existing ones are investigated. To emphasize the importance of ordered position of each argument and the importance of the argument itself, we also proposed the IVDHFEHA operator and IVDHFEHG operator, respectively. In addition, we put forward an approach to deal with MADM problems under intervalvalued dual hesitant fuzzy setting. Finally an illustrated example is given to show the developed method, and a comparison analysis is also conducted to demonstrate the effectiveness and superiority of the proposed approach. All the aggregation operators proposed in this paper are based on the assumption that the attributes in a given set are independent; that is, we only consider the addition of the importance of individual elements. However, in many practical situations, the elements in a set are usually correlative. Therefore, how to deal with the situations in which the arguments in a question are correlative is our future work.

\section{Conflict of Interests}

The authors declare that there is no conflict of interests regarding the publication of this paper.

\section{Acknowledgments}

The authors would like to express appreciation to the anonymous reviewers and the editor Wudhichai Assawinchaichote for their very helpful comments that improved the paper. This research is supported by Program for New Century Excellent Talents in University (NCET-13-0037), Natural Science Foundation of China (nos. 70972007, 71271049), and Beijing Municipal Natural Science Foundation (nos. 9102015, 9133020).

\section{References}

[1] L. A. Zadeh, "Fuzzy sets," Information and Computation, vol. 8, pp. 338-353, 1965.

[2] L. A. Zadeh, "The concept of a linguistic variable and its application to approximate reasoning. Parts 1, 2 and 3," Information Sciences, vol. 9, no. 1, pp. 43-80, 1975.

[3] D. Dubois and H. Prade, Fuzzy Sets and Systems: Theory and Applications, Academic Press, New York, NY, USA, 1980.

[4] K. T. Atanassov, "Intuitionistic fuzzy sets," Fuzzy Sets and Systems, vol. 20, no. 1, pp. 87-96, 1986. 
[5] K. Atanassov and G. Gargov, "Interval valued intuitionistic fuzzy sets," Fuzzy Sets and Systems, vol. 31, no. 3, pp. 343-349, 1989.

[6] Z. S. Xu, "A method based on linguistic aggregation operators for group decision making with linguistic preference relations," Information Sciences, vol. 166, no. 1-4, pp. 19-30, 2004.

[7] V. Torra and Y. Narukawa, "On hesitant fuzzy sets and decision," in Proceedings of the IEEE International Conference on Fuzzy Systems, pp. 1378-1382, Jeju Island, Republic of Korea, August 2009.

[8] V. Torra, "Hesitant fuzzy sets," International Journal of Intelligent Systems, vol. 25, no. 6, pp. 529-539, 2010.

[9] M. Xia and Z. Xu, "Hesitant fuzzy information aggregation in decision making," International Journal of Approximate Reasoning, vol. 52, no. 3, pp. 395-407, 2011.

[10] M. M. Xia, Z. S. Xu, and N. Chen, "Some hesitant fuzzy aggregation operators with their application in group decision making," Group Decision and Negotiation, vol. 22, no. 2, pp. 259-279, 2013.

[11] B. Zhu, Z. Xu, and M. Xia, "Hesitant fuzzy geometric Bonferroni means," Information Sciences, vol. 205, pp. 72-85, 2012.

[12] G. Wei, "Hesitant fuzzy prioritized operators and their application to multiple attribute decision making," Knowledge-Based Systems, vol. 31, pp. 176-182, 2012.

[13] Z. Zhang, "Hesitant fuzzy power aggregation operators and their application to multiple attribute group decision making," Information Sciences, vol. 234, no. 10, pp. 150-181, 2013.

[14] R. Lin, X. F. Zhao, and G. X. Wei, "Models for selecting an ERP system with hesitant fuzzy linguistic information," Journal of Intelligent Fuzzy Systems, vol. 26, no. 5, pp. 2155-2165, 2014.

[15] Z. Xu and M. Xia, "Distance and similarity measures for hesitant fuzzy sets," Information Sciences, vol. 181, no. 11, pp. 2128-2138, 2011.

[16] N. Chen, Z. Xu, and M. Xia, "Correlation coefficients of hesitant fuzzy sets and their applications to clustering analysis," Applied Mathematical Modelling: Simulation and Computation for Engineering and Environmental Systems, vol. 37, no. 4, pp. 2197-2211, 2013.

[17] Z. S. Xu and M. M. Xia, "Hesitant fuzzy entropy and crossentropy and their use in multiattribute decision-making," International Journal of Intelligent Systems, vol. 27, no. 9, pp. 799-822, 2012.

[18] B. Farhadinia, "Information measures for hesitant fuzzy sets and interval-valued hesitant fuzzy sets," Information Sciences, vol. 240, no. 10, pp. 129-144, 2013.

[19] N. Zhang and G. W. Wei, "A multiple criteria hesitant fuzzy decision making with Shapley value-based VIKOR method," Journal of Intelligent Fuzzy Systems, vol. 26, no. 2, pp. 1065-1075, 2014.

[20] Z. Xu and M. Xia, "On distance and correlation measures of hesitant fuzzy information," International Journal of Intelligent Systems, vol. 26, no. 5, pp. 410-425, 2011.

[21] X. Gu, Y. Wang, and B. Yang, "A method for hesitant fuzzy multiple attribute decision making and its application to risk investment," Journal of Convergence Information Technology, vol. 6, no. 6, pp. 282-287, 2011.

[22] X. Wang, Z. Gao, X. Zhao, and G. Wei, "Model for evaluating the government archives website's construction based on the GHFHWD measure with hesitant fuzzy information," International Journal of Digital Content Technology and its Applications, vol. 5, no. 12, pp. 418-425, 2011.
[23] Z. Liu and G. W. Wei, "An approach to evaluating the teaching quality of university with hesitant triangular fuzzy information," International Journal of Digital Content Technology and Its Application, vol. 7, no. 2, pp. 677-685, 2013.

[24] J. Ye, "Correlation coefficient of dual hesitant fuzzy sets and its application to multiple attribute decision making," Applied Mathematical Modelling, vol. 38, no. 2, pp. 659-666, 2014.

[25] Y. F. Chen, X. D. Peng, G. H. Guan, and H. D. Jiang, "Approaches to multiple attribute decision making based on the correlation coefficient with dual hesitant fuzzy information," Journal of Intelligent and Fuzzy Systems, vol. 26, no. 5, pp. 2547-2556, 2014.

[26] B. Zhu and Z. S. Xu, "Some results for dual hesitant fuzzy sets," Journal of Intelligent and Fuzzy Systems, vol. 26, no. 4, pp. 16571668, 2014.

[27] B. Zhu, Z. Xu, and M. Xia, "Dual hesitant fuzzy sets," Journal of Applied Mathematics, vol. 2012, Article ID 879629, 13 pages, 2012.

[28] H. J. Wang, X. F. Zhao, and G. W. Wei, "Dual hesitant fuzzy aggregation operators in multiple attribute decision making," Journal of Intelligent \& Fuzzy Systems, vol. 26, no. 5, pp. 22812290, 2014.

[29] Y. B. Ju, X. Y. Liu, and S. H. Yang, "Interval-valued dual hesitant fuzzy aggregation operators and their applications to multiple attribute decision making," Journal of Intelligent \& Fuzzy Systems, 2013.

[30] X. F. Zhao and G. W. Wei, "Some intuitionistic fuzzy Einstein hybrid aggregation operators and their application to multiple attribute decision making," Knowledge-Based Systems, vol. 37, pp. 472-479, 2013.

[31] W. Wang and X. Liu, "Intuitionistic fuzzy geometric aggregation operators based on einstein operations," International Journal of Intelligent Systems, vol. 26, no. 11, pp. 1049-1075, 2011.

[32] W. Wang and X. Liu, "Intuitionistic fuzzy information aggregation using einstein operations," IEEE Transactions on Fuzzy Systems, vol. 20, no. 5, pp. 923-938, 2012.

[33] W. Wang and X. Liu, "Interval-valued intuitionistic fuzzy hybrid weighted averaging operator based on Einstein operation and its application to decision making," Journal of Intelligent and Fuzzy Systems, vol. 25, no. 2, pp. 279-290, 2013.

[34] W. Z. Wang and X. W. Liu, “The multi-attribute decision making method based on interval-valued intuitionistic fuzzy Einstein hybrid weighted geometric operator," Computers \& Mathematics with Applications, vol. 66, no. 10, pp. 1845-1856, 2013.

[35] S. Zhang and D. J. Yu, "Some geometric Choquet aggregation operators using Einstein operations under intuitionistic fuzzy environment," Journal of Intelligent \& Fuzzy Systems, vol. 26, no. 1, pp. 491-500, 2014.

[36] X. F. Zhao, Q. X. Li, and G. W. Wei, "Model for multiple attribute decision making based on the Einstein correlated information fusion with hesitant fuzzy information," Journal of Intelligent \& Fuzzy Systems, vol. 26, no. 6, pp. 3057-3064, 2014.

[37] G. Wei and X. Zhao, "Induced hesitant interval-valued fuzzy Einstein aggregation operators and their application to multiple attribute decision making," Journal of Intelligent and Fuzzy Systems, vol. 24, no. 4, pp. 789-803, 2013.

[38] X. F. Zhao, R. Lin, and G. W. Wei, "Induced hesitant intervalvalued fuzzy Einstein aggregation operators and their application to multiple attribute decision making," Journal of Intelligent and Fuzzy Systems, vol. 41, no. 4, pp. 1086-1094, 2014.

[39] Z. Xu, "Intuitionistic fuzzy aggregation operators," IEEE Transactions on Fuzzy Systems, vol. 15, no. 6, pp. 1179-1187, 2007. 
[40] Z. S. Xu and R. R. Yager, "Some geometric aggregation operators based on intuitionistic fuzzy sets," International Journal of General Systems, vol. 35, no. 4, pp. 417-433, 2006.

[41] E. P. Klement, R. Mesiar, and E. Pap, "Triangular norms. Position paper. I. Basic analytical and algebraic properties," Fuzzy Sets and Systems, vol. 143, no. 1, pp. 5-26, 2004.

[42] Z. Xu, "On consistency of the weighted geometric mean complex judgement matrix in AHP," European Journal of Operational Research, vol. 126, no. 3, pp. 683-687, 2000.

[43] R. R. Yager, "On ordered weighted averaging aggregation operators in multicriteria decisionmaking," IEEE Transactions on Systems, Man, and Cybernetics, vol. 18, no. 1, pp. 183-190, 1988.

[44] Z. S. Xu and Q. L. Da, "The ordered weighted geometric averaging operators," International Journal of Intelligent Systems, vol. 17, no. 7, pp. 709-716, 2002.

[45] Z. S. Xu, Uncertain Multiple Attribute Decision Making: Methods and Applications, Tsinghua Uuniversity Press, Beijing, China, 2004. 


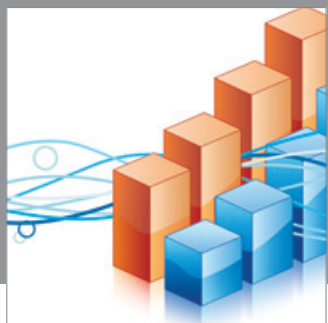

Advances in

Operations Research

mansans

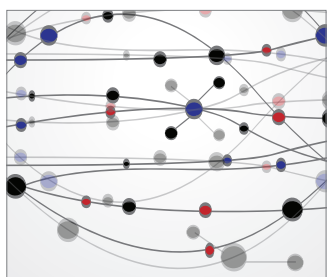

The Scientific World Journal
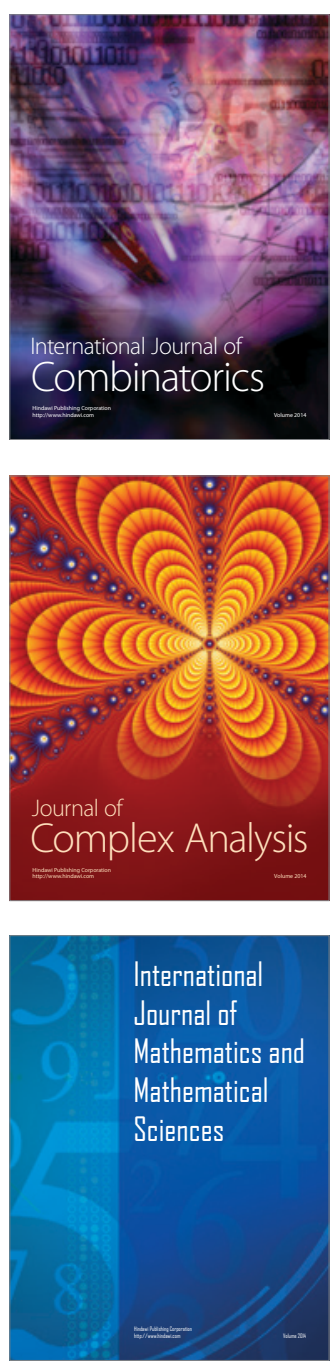
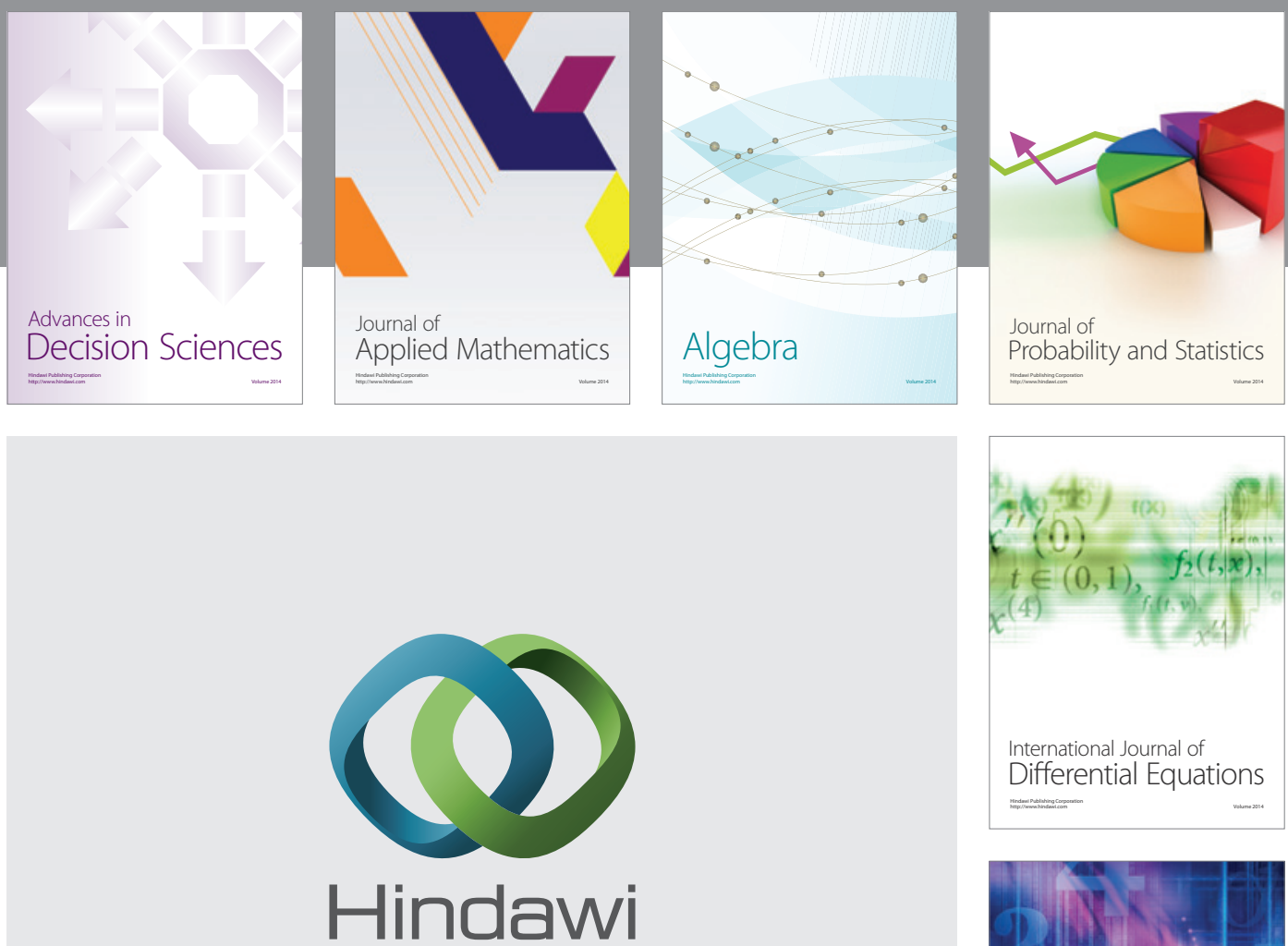

Submit your manuscripts at http://www.hindawi.com
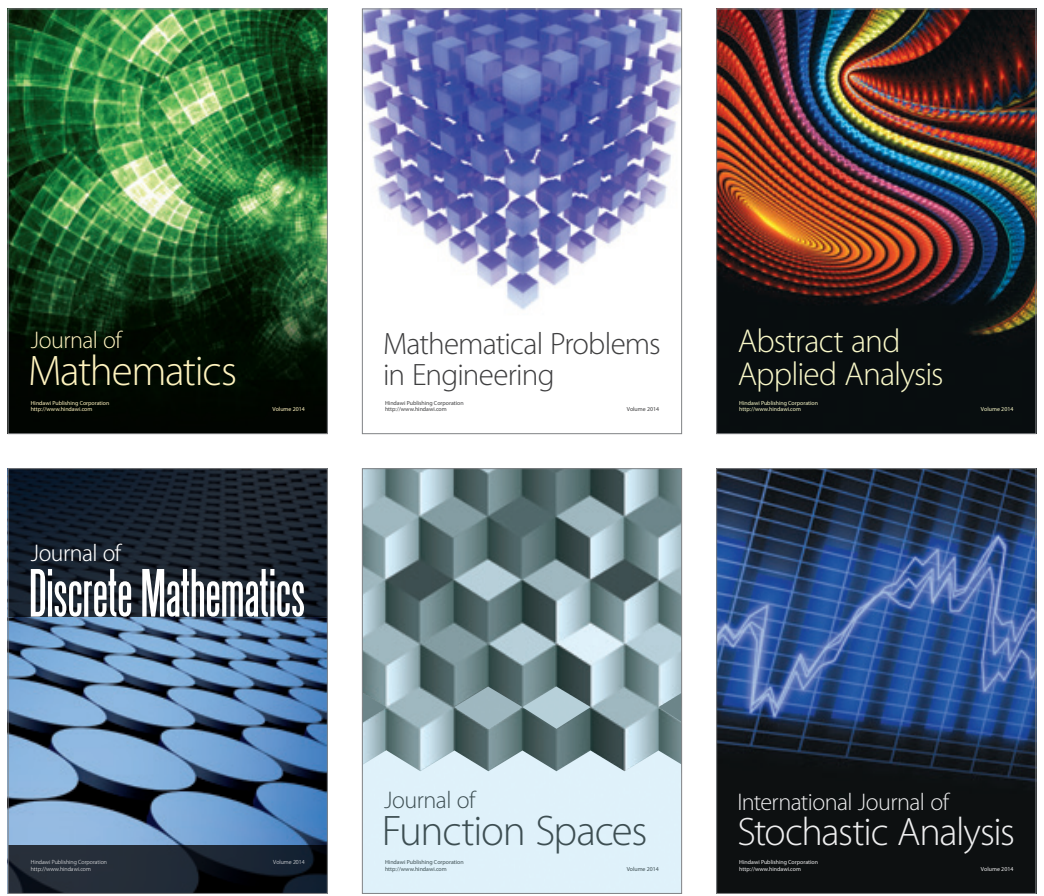

Journal of

Function Spaces

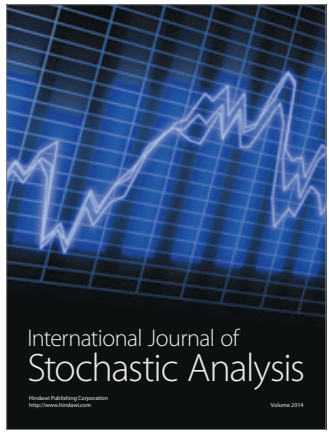

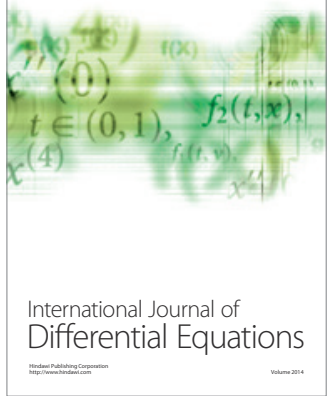
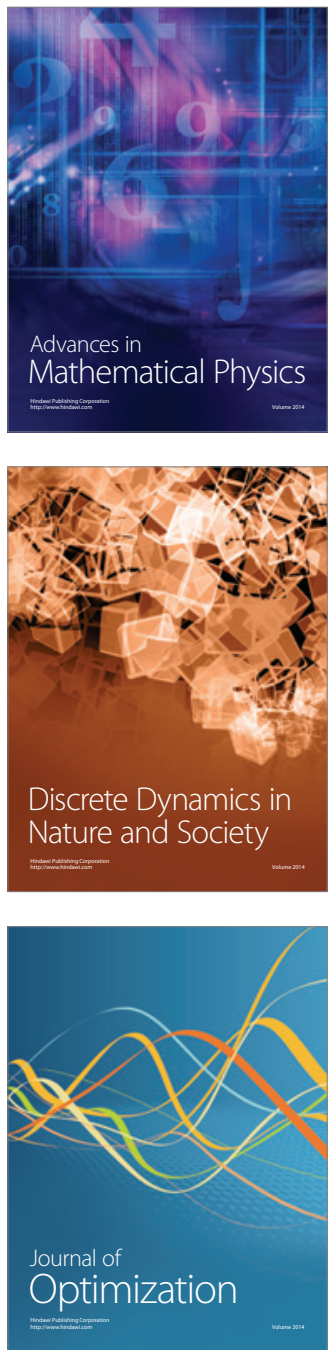